\title{
Pulsed-jet propulsion of a squid-inspired swimmer at high Reynolds number
}

\author{
Yang Luo a , Qing Xiao a,*, Qiang Zhu ${ }^{\mathrm{b}}$, Guang Pan ${ }^{\mathrm{c}}$ \\ aDepartment of Naval Architecture, Ocean and Marine Engineering, University of Strathclyde, \\ Glasgow, G4 0LZ, United Kingdom \\ bDepartment of Structural Engineering, University of California, San Diego, La Jolla, CA 92093, \\ United States of America

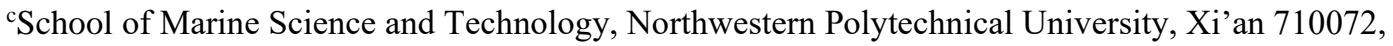 \\ People's Republic of China
}

\begin{abstract}
An inflation-deflation propulsion system inspired by the jet propulsion mechanism of squids and other cephalopods is proposed. The two-dimensional squid-like swimmer has a flexible mantle body with a pressure chamber and a nozzle which serves as the inlet and outlet of water. The fluid-structure interaction simulation results indicate that larger mean thrust production and higher efficiency can be achieved in high Reynolds number scenarios compared with the cases in laminar flow. The improved performance at high Reynolds number is attributed to stronger jet-induced vortices and highly suppressed external body vortices which are associated with drag force. Optimal efficiency is reached when the jet vortices start to dominate the surrounding flow. The mechanism of symmetry-breaking instability under the turbulent flow condition is found to be different from that previously reported in laminar flow. Specifically, this instability in turbulent flow stems from irregular internal body vortices, which causes symmetry breaking in the wake. Higher Reynolds number or smaller nozzle size would accelerate the formation of this symmetry-breaking instability.
\end{abstract}

\section{Introduction}

Aquatic organisms provide plentiful prototypes for artificial underwater vehicles and robots in terms of locomotion mechanism. Biological locomotion is categorized into three main types, fin oscillation, fin undulation and jet propulsion (Salazar et al., 2018). Extensive research focuses on the former two mechanisms in the literature. Compared with jet propulsion, locomotion by fin oscillation or undulation is more complicated. For example, bony fish fins

\footnotetext{
* Corresponding author: qing.xiao@strath.ac.uk
} 
are characterized by a soft collagenous membrane supported by embedded rays. These rays consist of two hemitrichs actuated by muscles at the base (Lauder et al., 2011). Although multiple degrees of freedom motion can be achieved by these composite structures, they inevitably increase the complexity in the design and manufacture of bio-inspired underwater vehicles. In comparison, jet propulsion employed by squids and other cephalopods is simpler and more straightforward to duplicate. The procedure starts with inflation of the mantle, a muscular organ that encloses the mantle cavity and the viscera (Bartol et al., 2008). During inflation, the internal volume of the cavity increases so that water fills the mantle cavity through the mantle aperture. This inflation was termed an inhalant phase or recovery stroke by Ward (1972). Afterwards, the squid contracts the mantle and the volume of the mantle cavity begins to decrease. Subsequently, water is ejected through the funnel tube (Fig. 1(a)) as a fast jet during the exhalant or power stroke phase to propel the squid in the opposite direction. Through this propulsion mechanism, effective escape locomotion and high manoeuvrability can be achieved (O’Dor, 2013). Some small squids, e.g., the larvae of Loligo vulgaris, are capable of bursting as fast as 25 body lengths per second (Gosline and DeMont, 1985).

(a)

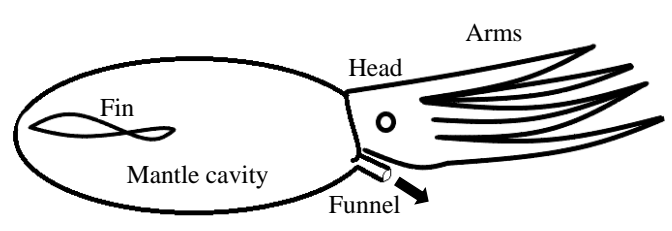

(b)

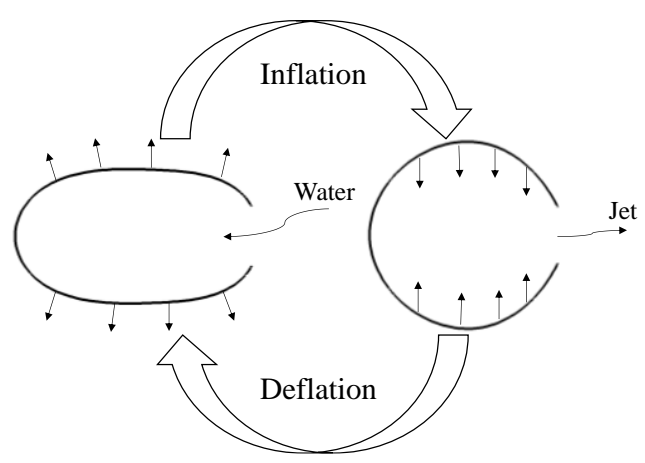

Fig. 1. The sketch of the structure of a squid (a) and the deflation-inflation cycle (b).

The jet propulsion mechanism of cephalopods bears certain resemblance to that of jellyfish, which has been broadly studied in the literature (Hoover et al., 2017; Megill et al., 2005; Miles and Battista, 2019; Park et al., 2015). Extensive advances in understanding propulsive mechanics derived from jellyfish models were summarized in a recent review paper (Costello et al., 2020). Nevertheless, as suggested by Bi and Zhu (2019b), it is not appropriate to apply the knowledge from jellyfish swimming to squids directly due to some critical differences 
between them. Specifically, jellyfish mainly rely on bell constriction to swim. They do not have a well-formed pressure cavity and a funnel which serves as a nozzle to separate the internal and external flows. Since jellyfish have limited capability to compress the entrained fluid, the jet flow they create is much weaker, and the instability issue may not be as pronounced as in the squid-created wake (Bi and Zhu, 2019b). Besides, different body geometry and kinematics also yield different vorticity control mechanism, which can be found from the comparison between Hoover and Miller (2015) and Bi and Zhu (2019b).

In contrast to the numerous existing studies of jellyfish swimming, studies on the jet propulsion of squids and other cephalopods are relatively few. Anatomical studies are important in improving our knowledge about the morphological and physiological basis of squid jet propulsion. For instance, experiments indicated that squid mantle contains two predominant muscle groups, circumferential muscle fibres or so-called circular muscles which constitute the bulk of the mantle wall, and radial muscles that extend from the inner to the outer surface of the mantle wall (Thompson and Kier, 2006). Detailed mantle kinematics were recorded during the swimming of Lolligunculu brevis (Ward, 1972). Furthermore, the activity patterns of these two groups of muscles were reported in Gosline et al. (1983), in which the hyper-inflation of the mantle powered by the radial muscles, the deflation powered by circular muscles, and the refilling powered mainly by the radial muscles were observed. In addition, jet hydrodynamics and flow patterns of live squids were studied by using digital particle imaging velocimetry (DPIV) techniques (Anderson and Grosenbaugh, 2005; Bartol et al., 2016; Bartol et al., 2009a; Bartol et al., 2008). These studies illustrated the near-body flow field and the evolution of ejected vortex rings.

Based on the above biological studies, some prototype underwater vehicles or robots have been designed and manufactured. For example, a cephalopod-inspired soft unmanned underwater vehicle (SUUV) with cable-driven pulsed-jet propulsion was reported (Renda et al., 2015). In another experiment by Weymouth et al. (2015), a more true-to-nature octopus-inspired flexible hull robot showed excellent fast-starting performance comparable to its natural counterpart. However, it lacked a refillable pressure chamber, so that it can only perform one burst-coast motion. A recent study proposed a novel jet-propelled squid-like prototype with soft pneumatic 
morphing design (Hou et al., 2019). To shuttle between water and air, the jet was powered by compressed air in the gas chamber instead of ejected water used by squids.

Besides those experimental studies, a handful of theoretical studies have been conducted to elucidate the underlying mechanism of jet propulsion. A simple shape-changing body was demonstrated to be able to hover or ascend in an oscillating background flow by Spagnolie and Shelley (2009), and the achieved velocity burst was thought to be related to the escape dynamics of aquatic invertebrates. Subsequent studies revealed the significance of added-mass energy recovery in the bursting motion of shape-changing bodies (Steele et al., 2017; Weymouth and Triantafyllou, 2013). It was illustrated that considerable thrust augmentation, almost comparable to the jet-generated thrust, can be obtained from the recovery of added-mass energy as the body deflates. Nevertheless, successful energy recovery depends highly on the body shrinking speed and Reynolds number. In these studies, the body kinematics were prescribed, and the interaction between the flexible body and surrounding fluid was not considered. Additionally, the internal cavity flow was excluded.

Following these studies, a series of numerical simulations have been conducted by Bi and Zhu $(2018,2019 \mathrm{a}, 2019 \mathrm{~b}, 2020)$ to understand the combined effect of jetting and body deformation. By using a three-dimensional boundary-element method, they investigated the burst-coast swimming of a cephalopod-like deformable body with a pressure chamber. Two-dimensional fluid-structure interaction (FSI) model based on the immersed-boundary framework was developed subsequently to study the fully-viscous response of the squid-like jet propulsion system activated by a sequence of springs in tethered (Bi and Zhu, 2019b) and free-swimming (Bi and Zhu, 2019a) modes under low Reynolds number conditions. Symmetry-breaking instability and effect of added-mass on the jet propulsion were demonstrated.

In this work, we numerically study a squid-like jet propulsion system with a more realistic activation mechanism in high Reynolds number (turbulent flow) conditions. As shown in Fig. 1(b), an external forcing which mimics the constriction of the circular muscles is introduced to actuate the deflation of the mantle body. The re-expansion is driven by the release of the stored elastic energy in the mantle structure. By performing FSI study at high Reynolds numbers, our purpose is to explore how the flexible mantle structure would behave and whether pulsed thrust 
would be produced through the interaction with the surrounding medium. With turbulent flow being considered, we aim at examining to what extent the jet thrust would be augmented compared with the laminar flow scenarios as studied by Bi and Zhu (2019b). Besides, it is interesting to investigate how the symmetry-breaking instability which has been demonstrated in Bi and Zhu (2019a) would develop in turbulent flow condition.

In comparison with the two-dimensional numerical study of a similar system (Bi and Zhu (2019b), the novelty of the present study lies in two aspects. Firstly, similar to most existing numerical studies of squid-like swimming, the work by Bi and Zhu (2019b) was limited to the laminar flow regime. They only covered the flow regimes $(\operatorname{Re}=1 \sim 100)$ where squid hatchlings operate, while juveniles and adults swim at much higher Reynolds numbers $\left(10^{3} \sim 10^{6}\right)$. Reynolds number has been proven to play a non-negligible role in the hydrodynamics of squids (Bartol et al., 2009b; Bartol et al., 2008). Besides, it is difficult to directly employ numerical results in laminar flow to guide the design of squid-inspired robots or vehicles which operate at much higher Reynolds numbers (Christianson et al., 2020). Secondly, our actuation mechanism is more biologically relevant. In our model, the function of the circular muscles of the squid mantle is mimicked, and the inflation is powered by the elastic energy of the mantle structure, which is consistent with the activation mechanism of squid mantle. In contrast, the inflation-deflation pattern in Bi and Zhu (2019a, 2019b) was achieved by the variation of lengths of virtual springs inside the cavity along the longitudinal direction. The strain energy in these springs, instead of that in the mantle structure, activated the system.

The rest of this study is organized as follows. The geometry and actuation mechanism of the squid-like swimmer model is introduced in Section 2. These are followed by the governing equations and numerical approaches in Section 3. Afterwards, numerical results are presented. Finally, conclusions and discussion are summarized in Section 5.

\section{Problem Statement}

\subsection{Squid-like swimmer model}

A two-dimensional swimmer inspired by the jet propulsion mechanism of squid is considered. It is modelled as an elliptical shell whose right end is removed to form the nozzle. The 
simplification of the squid-like swimmer as a hemi-ellipsoid has been found in previous studies (e.g., (Bi and Zhu, 2019b; Hoover and Miller, 2015)). The initial geometry of the body before deformations, as shown in Fig. 2, is given by

$$
\frac{x^{2}}{a^{2}}+\frac{y^{2}}{b^{2}}=1, \text { for } x \leq c,
$$

where $a$ is the length of the body at the left half, $b$ is the body height and $c$ is the length of the body at the right half.

As shown in Fig. 1(b), the body undergoes deflation and inflation during one jet cycle, similar to that observed in squid swimming. As mentioned earlier, the jet propulsion of the squid involves two groups of muscles, radial and circular, as well as the mantle wall. The radial muscles are usually active during the escape or predation manoeuvre for hyperinflation of the mantle cavity. The contraction of the circular muscles actuates the deflation for the jetting. During regular swimming, the expansion is mostly powered by the elastic recoil of the mantle wall. Inspired by this mechanism, in this study the deformation of the mantle consists of two phases, the active deflation which is actuated by the applied external force mimicking the function of the circular muscles, and the passive inflation resulting from release of the elastic energy in the mantle structure. During the inflation phase, the enclosed area increases so that fluid enters through the nozzle (the opening of the ellipse with size $R$ ). Afterwards, the fluid is expelled via the nozzle when the internal area is decreased during the deflation. To drive the motion of the body, an external forcing term, $F_{\text {ef }}$, is applied to the body surface in the transverse direction, as presented in Fig. 2, which is given by

$$
F_{\text {ef }}(t)=\left\{\begin{array}{l}
F_{\text {ef0 }} \sin \left(2 \pi f_{\text {ef }} t\right), t<=n T_{\mathrm{i}}+1 / 2 T_{\text {ef }} \\
0, t>n T_{\mathrm{i}}+1 / 2 T_{\text {ef }}
\end{array},\right.
$$

where $n$ is a non-negative integer $(n=0,1,2,3 \ldots), T_{\text {ef }}=1 / f_{\text {ef }}$ is the period of the external forcing term with $f_{\text {ef }}$ the actuation frequency, $t$ is time, and $T_{\mathrm{i}}$ is the time it takes for the passive inflation of the body obtained from the free vibration study (see Appendix B). The amplitude of the external force is $F_{\text {ef } 0}=0.5 \rho_{f} U^{2} L \cdot C_{\text {ef } 0}$, where $\rho_{f}$ is the density of the fluid, $U$ is the free stream velocity, $L$ is the contour length of the body with medium nozzle size ( 
$R=0.1 L)$ and $C_{\text {ef } 0}$ is the external force coefficient. The complete locomotion period, including inflation and deflation, is $T=0.5 T_{\text {ef }}+T_{\mathrm{i}}$.
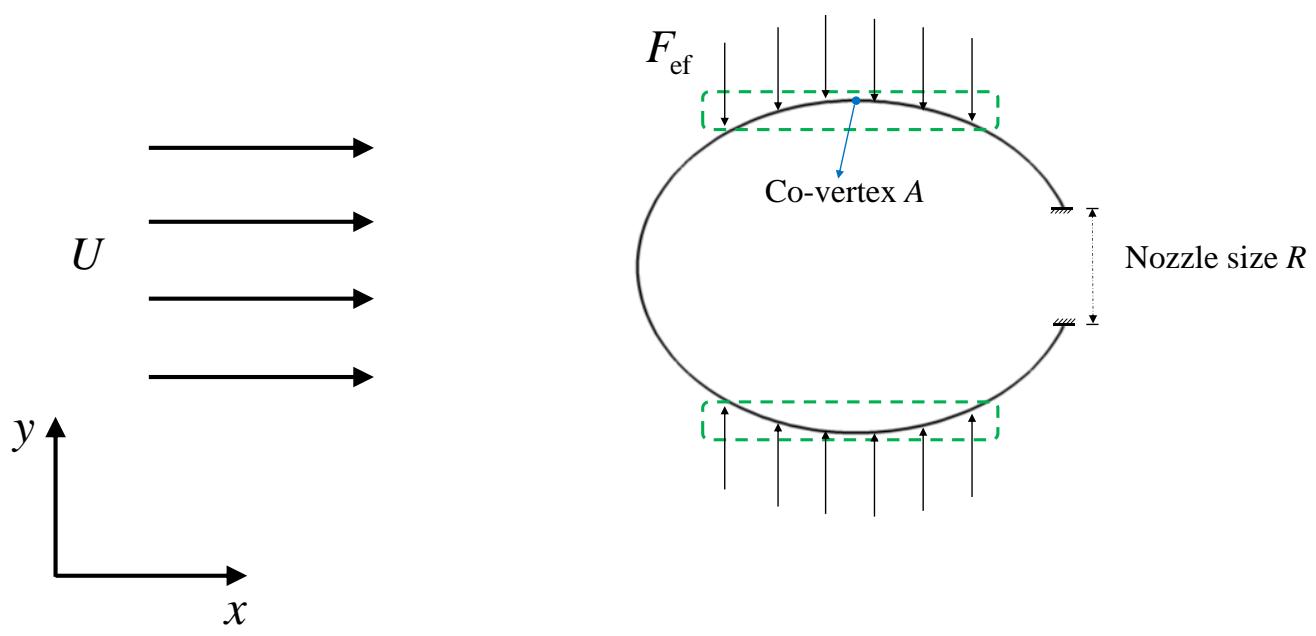

Fig. 2. The external force is evenly distributed on the mesh nodes in the green rectangular zone with left boundary at $x=-0.78 a$ and right boundary at $x=0.78 a . U$ is the free stream velocity and $F_{\text {ef }}$ denotes the applied external force.

The dimensionless parameters governing the problems are defined as: the Reynolds number $\operatorname{Re}=L U / v$ with $v$ the kinematic viscosity of the fluid; the dimensionless frequency (the Strouhal number) of the external force $S t=f_{\mathrm{ef}} L / U$; the mass ratio $m^{*}=\rho_{s} h / \rho_{f} L$ with $h$ denoting the thickness of the mantle and $\rho_{s}$ the density of the structure; the non-dimensional stiffness $K=E I /\left(\rho_{f} U^{2} L^{3}\right)$, where $E$ is Young's modulus and $I=h^{3} / 12$ represents the area moment of inertia of the cross-section; and the Poisson ratio $v_{s}$.

\subsection{Performance metrics}

By integrating the distributed fluidic stress along the swimmer body, we obtain the instantaneous thrust coefficient as

$$
C_{T}=-\frac{F_{x}}{\frac{1}{2} \rho_{f} U^{2} L},
$$


where $F_{x}$ is the component of the hydrodynamic force in the $x$-direction.

The instantaneous power expenditure coefficient is computed by

$$
C_{P}=\frac{P_{\mathrm{ef}}}{\frac{1}{2} \rho_{f} U^{3} L},
$$

where $P_{\text {ef }}$ is the total power output of the external force, which is calculated as $P_{\text {ef }}=\int \boldsymbol{F}_{\text {ef }} \bullet \boldsymbol{u} d s$ with $\boldsymbol{u}$ the velocity of the grid node $s$ where the external force is applied. In the tethered case with a constant incoming fluid velocity, the propulsive efficiency is defined as the ratio between the mean thrust coefficient and the mean power expenditure coefficient so that we have

$$
\eta=\frac{\overline{C_{T}}}{\overline{C_{P}}} .
$$

\section{Numerical model and method}

The numerical solver involves the resolution of fluid and structural dynamics. The dynamic interplay between the fluid and the structure is resolved in a partitioned manner.

The turbulent flow around the swimmer is governed by unsteady, compressible and viscous Navier-Stokes equations with the two-equation $k-\omega$ model by Wilcox (1998). Under the assumption of no internal source term and by ignoring the body force, the governing equations can be written in an integral form as

$$
\frac{\partial}{\partial t} \iiint_{\Omega_{f}} \mathbf{W} d \Omega_{f}+\iint_{S_{f}} \mathbf{G} d S_{f}=0,
$$

where $\mathbf{W}=\left(\rho_{f}, \rho_{f} \boldsymbol{v}, \rho_{f} E, k, \omega\right)^{T}$ is the conservative variable vector, with $\rho_{f}$ the fluid density, $v$ the velocity vector in the Cartesian coordinate system, $E$ the total energy, $k$ the turbulent mixing energy, and $\omega$ the specific dissipation rate. $\Omega_{f}$ is the fluid control domain with the boundary as $S_{f}$. G is the flux vector which consists of two parts, the convective and pressure fluxes, and the fluxes arising from the viscous shear stress and thermal diffusion. To numerically solve the above governing equations, they are discretized using a cell-centred finite volume method. Specifically, the fluid domain $\Omega_{f}$ is divided into an array of hexahedral 
grid cells indexed by $(i, j, k)$. The governing equations are applied to each of these cells, and a semi-discretized form can be obtained as

$$
\frac{\partial}{\partial t}\left(\mathbf{W}_{i, j, k} \Delta \Omega_{f}\right)_{i, j, k}-\mathbf{R}_{i, j, k}=\mathbf{D}_{i, j, k},
$$

where $\Delta \Omega_{f}$ is the control volume of the cell $(i, j, k)$, and $\mathbf{R}_{i, j, k}$ is the residual measuring the convective and diffusive fluxes entering the hexahedral cell through its surface. An artificial viscosity term $\mathbf{D}_{i, j, k}$ is introduced to stabilize the scheme and eliminate the spurious numerical oscillations (Jameson et al., 1981).

The dual-time stepping algorithm is implemented for the current unsteady computation, in which Eq. (7) is reformulated as a steady-state flow problem with a pseudo-time $\hat{t}$

$$
\frac{\partial}{\partial \hat{t}} \mathbf{W}^{n+1}=\mathbf{R}^{*}\left(\mathbf{W}^{n+1}\right) / \Delta \Omega_{f},
$$

where

$$
\mathbf{R}^{*}\left(\mathbf{W}^{n+1}\right)=\mathbf{R}\left(\mathbf{W}^{n+1}\right)+\mathbf{D}\left(\mathbf{W}^{n+1}\right)-\frac{3\left(\mathbf{W} \Delta \Omega_{f}\right)^{n+1}-4\left(\mathbf{W} \Delta \Omega_{f}\right)^{n}+\left(\mathbf{W} \Delta \Omega_{f}\right)^{n-1}}{2 \Delta t},
$$

where the superscripts $n-1$ and $n$ denote the two previous time steps and this yields secondorder accuracy. Eq. (9) is then integrated using a hybrid multistage Runge-Kutta scheme. Within the numerical flow solver, parallel computation is enabled by domain decomposition via message passing interface (MPI) to achieve large-scale computation. Furthermore, the local time-stepping and multigrid method are implemented to accelerate the convergence. More detailed formulations of this fluid solver can be found in Sadeghi (2004).

It is worth noting that to simulate an incompressible flow problem using the present compressible flow solver, it is necessary to ensure that the compressibility is negligibly small. As we did in the previous applications to incompressible simulations, the freestream Mach number, defined as $M a_{\infty}=U / a_{\infty}$ with $a_{\infty}$ denoting the speed of sound, is chosen as 0.06 . This value is far below the critical value of 0.3 , where the compressibility effect becomes pronounced, but still sufficiently large to ensure numerical stability. Given the fact that the boundary is moving and flow is ejected during the motion of the mantle cavity, the actual Mach number may become larger than $M a_{\infty}$ near the swimmer. Therefore, for computation accuracy, 
the local Mach numbers in the whole computational domain are monitored during computation to ensure that they are always below the critical value. This compressible flow solver has been successfully applied to various incompressible flow simulations in our previous work (Liu et al., 2016; Luo et al., 2020a; Luo et al., 2020b; Shi et al., 2019; Xiao and Liao, 2010; Xiao et al., 2012).

Regarding the structural dynamics, the governing equation is the weak form of the balance of momentum which is written the differential form as

$$
\frac{D^{2} \mathbf{S}}{D^{2} t^{2}}=\frac{1}{\rho_{s}} \nabla \cdot \mathbf{P}+\mathbf{f},
$$

where the left part of the equation is the acceleration of the material point, which is calculated by the second derivatives of displacement vector $\mathbf{S}$ of the structure. The surface forces are modelled by the second Piola-Kirchoff stress tensor $\mathbf{P}$, and $\mathbf{f}$ denotes the body forces per unit mass.

To close up Eq. (10), a constitutive equation which describes the correlation between the stress and the strain is defined. Specifically, for a Saint Venant-Kirchhoff material, the second PiolaKirchoff stress tensor $\mathbf{P}$ is calculated by

$$
\mathbf{P}=\mathbf{C}: \mathbf{E}, \mathbf{E}=0.5\left(\mathbf{F}^{T} \mathbf{F}-\boldsymbol{\delta}\right),
$$

where $\mathbf{C}$ represents the elastic tensor, $\mathbf{E}$ is the Green-Lagrange strain tensor, $\mathbf{F}$ denotes the deformation gradient, and unit tensor is characterized by $\boldsymbol{\delta}$.

The present structural solver is based on CalculiX (Dhondt, 2004), in which the finite element method is employed to discretize the general governing equations Eq. (10). With the application of the virtual work method, a linear algebraic equation system by the discretization in the whole solid domain can be obtained as

$$
[\mathbf{K}]\{\mathbf{S}\}+[\mathbf{M}] \frac{D^{2}}{D t^{2}}\{\mathbf{S}\}=\{\mathbf{F}\}
$$

where the global stiffness matrix, the global mass matrix and the global force vector are denoted by $[\mathbf{K}],[\mathbf{M}]$ and $[\mathbf{F}]$ respectively. 
The temporal discretization is achieved by the utilization of the $\alpha-$ method described in Dhondt (2004). Denoting the velocity vector as $\{\mathbf{U}\}:=\{\dot{\mathbf{S}}\}$ and the acceleration vector as $\{\mathbf{A}\}:=\{\ddot{\mathbf{S}}\}$, we can obtain the solution at the time step $n+1$ by

$$
\begin{gathered}
\{\mathbf{U}\}^{n+1}=\{\hat{\mathbf{U}}\}^{n+1}+\gamma \Delta t\{\mathbf{A}\}^{n+1}, \\
\{\mathbf{S}\}^{n+1}=\{\hat{\mathbf{S}}\}^{n+1}+\beta(\Delta t)^{2}\{\mathbf{A}\}^{n+1},
\end{gathered}
$$

where $\{\hat{\mathbf{U}}\}^{n+1}$ and $\{\hat{\mathbf{S}}\}^{n+1}$ are the predictor at time step $n+1$, which are only dependant upon the values at the time step $n . \gamma$ and $\beta$ are constants determined by the value of $\alpha$.

In addition to the fluid and solid solvers, the coupling between them is another main ingredient for a partitioned FSI solver. In this work, the fluid solver and structural solver are coupled via preCICE (Bungartz et al., 2016), a coupling library for partitioned multi-physics simulations. Within this FSI solver, an implicit coupling scheme is designed, in which sub-iterations are introduced during each time step to ensure numerical stability and convergence. An improved IQN-ILS method is applied to stabilize and accelerate coupling iterations (Mehl et al., 2016). The details of this FSI solver and its validations are provided in Luo et al. (2020b). The builtin $k-\omega$ model has been successfully applied to the simulation of turbulent flow with $\operatorname{Re}$ number up to $\mathrm{O}\left(10^{6}\right)$ and validated via comparisons with previous numerical and experimental data in Appendix A and Sadeghi et al. (2003).

The computational domain is shown in Fig. 3(a). On the squid-like swimmer surface, the noslip/no-flux condition is applied, while for the upper and lower domain boundaries, the nonreflective far-field boundary condition is imposed. Velocity inlet and pressure outlet boundary conditions are imposed on the left and right domain boundaries, respectively. A selfconsistency study is performed to assess the appropriate mesh and time-step resolution by solving the turbulent flow for $\operatorname{Re}=U L / v=1 \times 10^{5}, m^{*}=0.05, K=0.05, C_{\mathrm{ef} 0}=0.64, S t=4.4$, and $R=0.1 L$. Three fluid meshes are generated: a coarse mesh with 60290 cells, a medium mesh with 78758 cells and a fine mesh 105220 cells. The height of the first cell is $1.98 \times 10^{-4} \mathrm{~L}$ to yield a $y^{+}$value 1 for the three grids. The structural mesh contains 198 quadratic brick elements. Firstly, the mesh convergence test is performed in which three fluid meshes are used 
along with the dimensionless time step size, defined as $\overline{\Delta t}=\Delta t U / L, \overline{\Delta t}=0.00113$. The discrepancy in $C_{T}$ among results obtained with the three meshes is shown in Fig. 4(a) in terms of relative error with the result of the fine mesh as reference. It can be seen that the medium mesh yields result very close to that of the fine mesh with the maximum difference between them less than $2 \%$. Thereafter, the effect of time step size when the medium mesh is used is also studied, as shown in Fig. 4(b). These tests show that the results are not sensitive to the mesh size and time step size if they are small enough. Therefore, to ensure the computation accuracy and meanwhile, reduce the computational cost, the medium mesh is used for the following simulations.

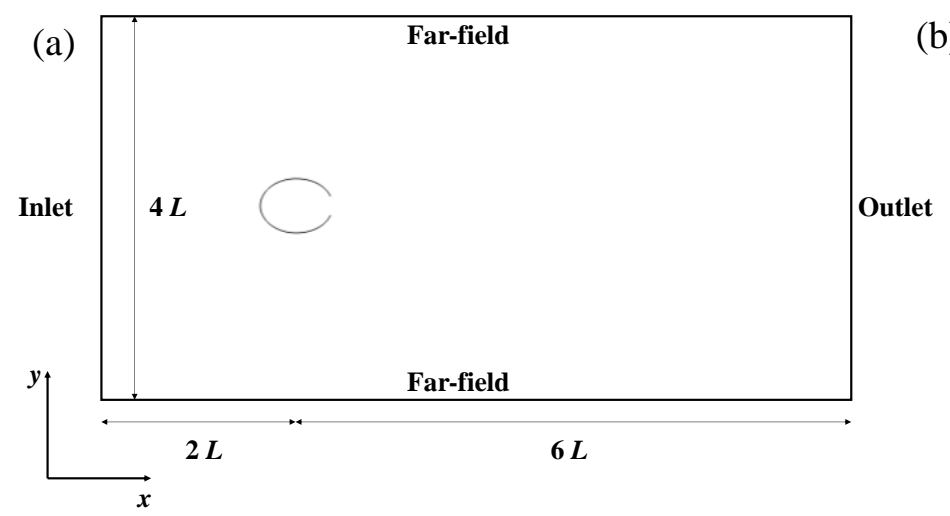

(b)

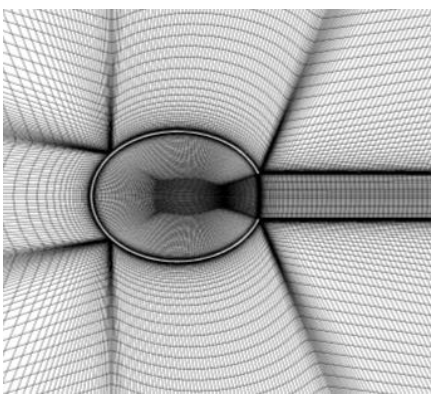

Fig. 3. The computational domain layout (a) and the generated medium size mesh around the swimmer (b) when the nozzle size $R=0.1 L$. 
(a)

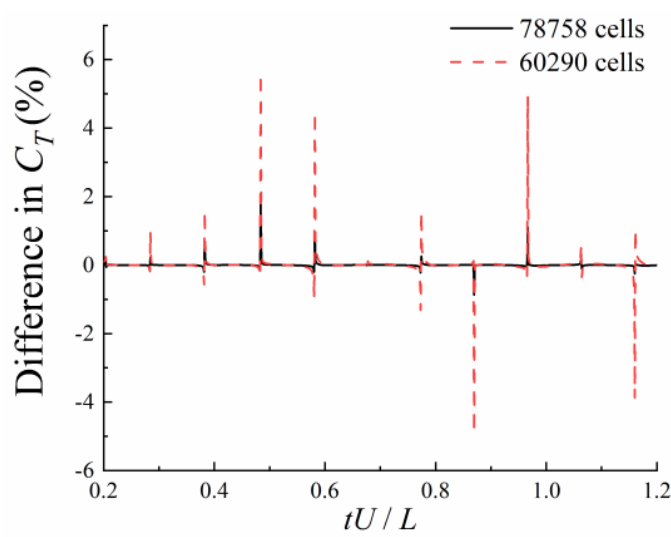

(b)

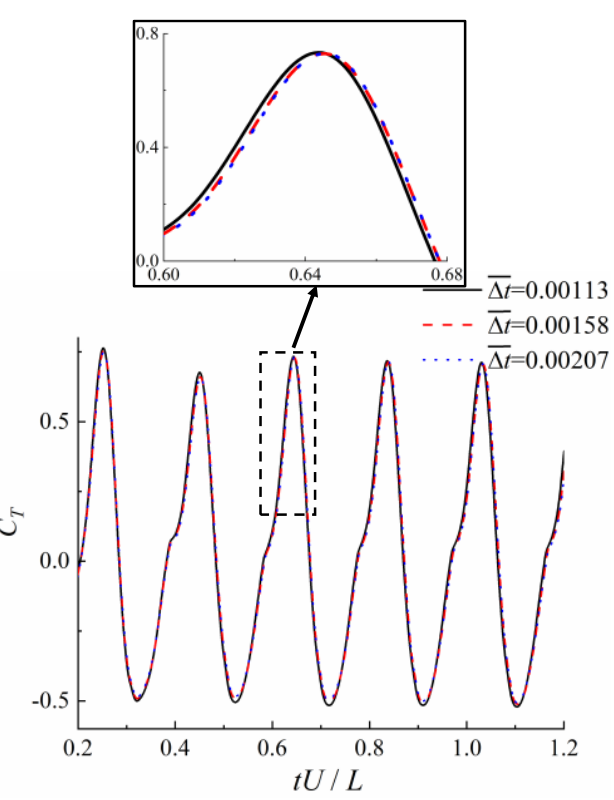

Fig. 4. The discrepancy in $C_{T}$ among results obtained with the three different fluid meshes when

$\overline{\Delta t}=0.00113$ with the results of the fine mesh with 105220 cells as reference (a), and comparison of $C_{T}$ for the medium size mesh when three different time step sizes are used (b).

\section{Results}

Numerical tests have been performed to determine the appropriate stiffness and external force amplitude after the self-consistency study of mesh and time step size. Our results indicate that under the combination of $K=0.05$ and $C_{\text {ef }}=0.64$, regular and periodic inflation-deflation locomotion is achievable and lasts sufficiently long to reach steady state. Meanwhile, the deformation is not too large to induce instability of the locomotion system, or too small so that no thrust is produced. Therefore, they are used in the following simulations in this study.

\subsection{Steady-state response}

The steady-state response of a typical case is presented to demonstrate the deflation-inflation cycle of the body and the resultant propulsive performance. In this simulation, the dimensionless parameters are listed in Table I. It is noted that when $\operatorname{Re}=1 \times 10^{5}$ and $K=0.05$, the free vibration frequency of the body is $S t_{\mathrm{v}}=6.3$ obtained from the free vibration study in 
Appendix B. The passive inflation period $T_{\mathrm{i}}$ is half of the free vibration period, i.e., $T_{\mathrm{i}}=0.5 \mathrm{~L} /\left(U S t_{\mathrm{v}}\right)$, as shown in Fig. 22. Therefore, the complete locomotion period is $T=0.5 L\left(1 / S t+1 / S t_{\mathrm{v}}\right) / U$.

Table I. List of the parameters used in the simulation.

\begin{tabular}{cccccccc}
\hline $\operatorname{Re}$ & $K$ & $C_{\text {ef0 }}$ & $S t$ & $m^{*}$ & $a$ & $b$ & $R$ \\
\hline $1 \times 10^{5}$ & 0.05 & 0.64 & 3.6 & 0.05 & $0.2 L$ & $0.15 L$ & $0.1 L$ \\
\hline
\end{tabular}

The change of body shape within one period is plotted in Fig. 5 to demonstrate the body deformation during the jet propulsion cycle. As can be seen, the deformation at this stiffness and external force frequency is not as noticeable as that in Bi and Zhu (2019b) (see Fig. 8 in their work) where the actuation mechanism is different from the current study. In that study, the inflation and deflation of the mantle wall were the results of the stretch and contraction of virtual lateral springs inside the cavity. By specifying the longitudinal position and stiffness of a single spring, they were able to control the deformation of the body along the longitudinal direction. However, the current deformation of the body is the response of the entire mantle structure to the external force, fluidic force and structural inertial force. Mathematically, it is the solution of the fully coupled fluid and structure equations in Eq. (6) and Eq. (10). This may explain the difference in body deformation between the present study and that in $\mathrm{Bi}$ and Zhu (2019b). Specifically, the deformation is smooth along the circumferential direction, as shown in Fig. 5 in this work, which is reminiscent of the deformation of a jellyfish-like swimmer model in Hoover and Miller (2015) where a similar actuation mechanism was employed. In contrast, the deformation is more complicated in the circumferential direction in Bi and Zhu (2019b) (as shown in Fig. 9 in their study).

To quantitatively record the locomotion of the mantle wall, we also depict the variation of the Y position of the co-vertex $A$ shown in Fig. 2 in Fig. 6(a). As aforementioned, one locomotion period consists of the active deflation under external force and the passive inflation from structural recoil. It can be seen that even though the external forcing term is still in place, the body starts to swell ahead of the middle of the period, indicating that the external force is not 
large enough to counter the rebound force at this instant. The maximum Y position is seen slightly ahead of the beginning of the next cycle of exerting the actuation force, which implies that the maximum inflation is reached under the free vibration condition.

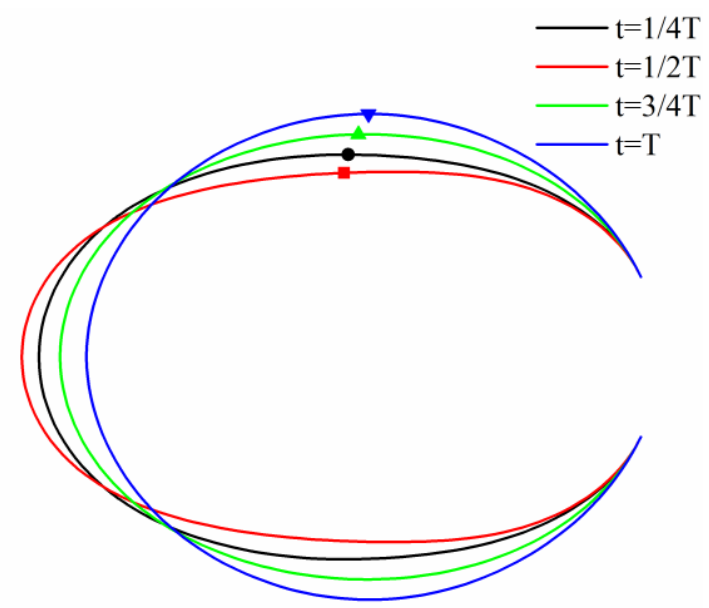

Fig. 5. The evolution of the body shape within one period. The co-vertex $A$ is marked by a circle ( $\mathrm{t}=1 / 4$ $\mathrm{T})$, a square $(\mathrm{t}=1 / 2 \mathrm{~T})$, an up triangle $(\mathrm{t}=3 / 4 \mathrm{~T})$ and a down triangle $(\mathrm{t}=\mathrm{T})$.

The variation of the thrust and jet speed is plotted in Fig. 6(b). A comparison of Fig. 6(a) and Fig. 6(b) reveals that most of the thrust is generated during the deflation phase, and the peak thrust occurs before the fully deflated status of the body. Although nearly half of a locomotion period is characterized by drag production, especially during the inflation phase, the mean thrust is still positive mainly due to a larger magnitude of maximum thrust than that of the maximum drag force. This feature is different from the fin-based propulsion mode, where a fish can almost avoid the creation of drag force by complex 3D conformations of the flexible fin (Han et al., 2020). Following Bi and Zhu (2019b), the average jet speed here is defined as $V_{j}=-\frac{1}{R} \frac{d S}{d t}$, where $S$ is the internal area whose variation is depicted in Fig. 6(d). Similar to the finding by Bi and Zhu (2019b), there is a phase lag between the thrust production and jet speed, as shown in Fig. 6(b). This may be due to the influence of drag force on the mantle body, the jet acceleration effect (Bi and $\mathrm{Zhu}, 2020$ ), and the added-mass related thrust (Bi and Zhu, 2019b). 
(a)

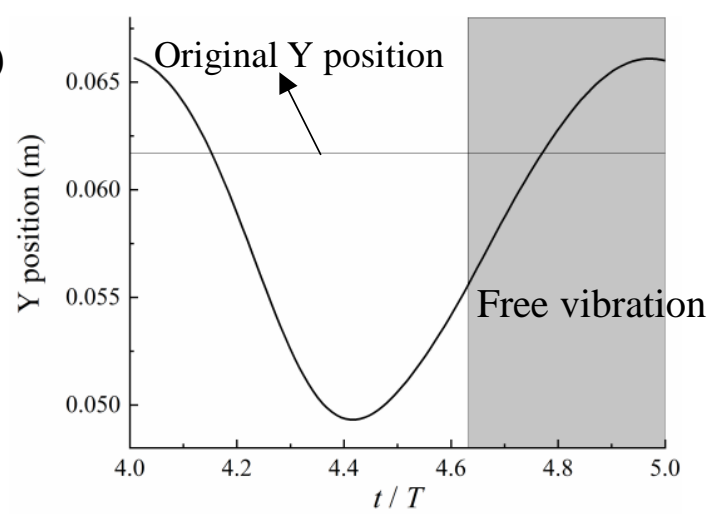

(b)

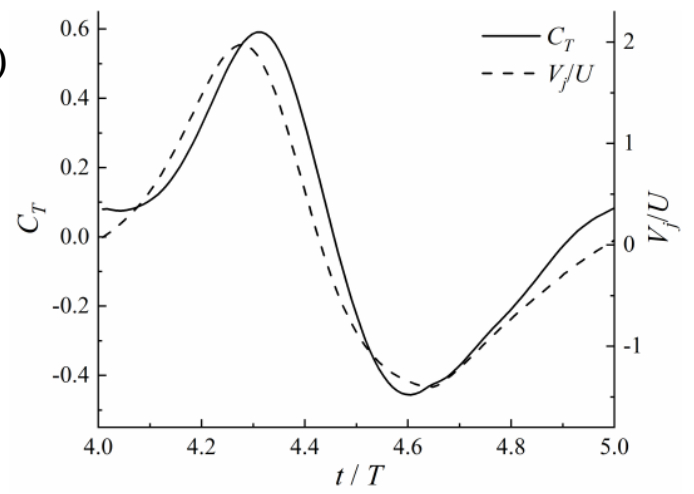

(c)

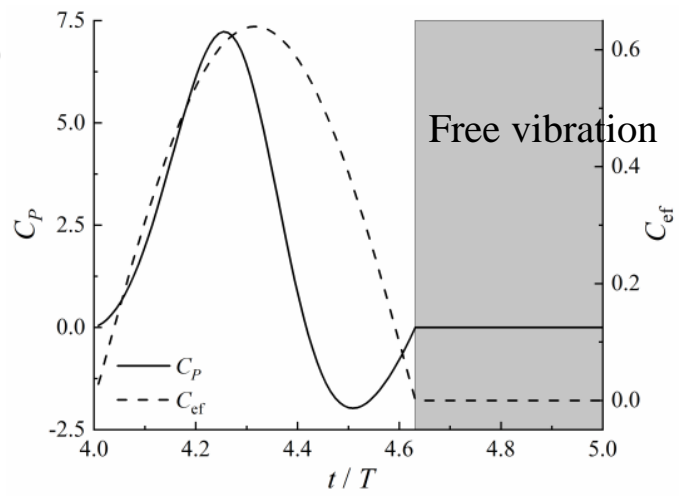

(d)

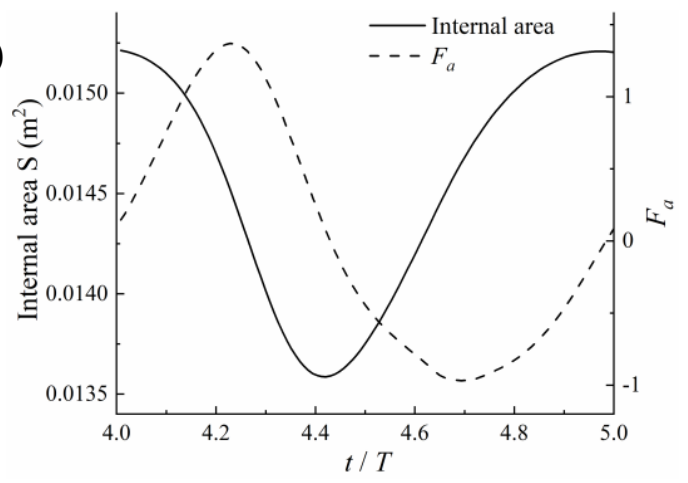

Fig. 6. Time history of the Y position of the co-vertex (a), the instantaneous thrust coefficient $C_{T}$ and jet speed (b), the instantaneous power expenditure coefficient $C_{P}$ and applied external force coefficient $C_{\text {ef }}$ (c) and the internal surface area and dimensionless added-mass-related thrust coefficient (d) within one period. The duration of free vibration is filled with grey background in (a) and (c). 
The power expenditure coefficient $C_{P}$ and applied instantaneous external force coefficient $C_{\text {ef }}$ are shown in Fig. 6(c). It can be observed that most of the actuated force does positive work unless near the end of the force-exerting phase when the structure recoils. The jet flow is directly associated with the internal area of the chamber, and the change of this body shape would induce added-mass-related thrust. Fig. 6(d) presents the variation of internal area and the added-mass-related thrust within one locomotion period. The dimensionless added-mass related thrust is determined by $F_{a}=-\dot{m}_{a} U / 0.5 \rho_{f} U^{2} L$ where $m_{a}$ is the added mass. The instantaneous shape of the body is simplified as an ellipse with semi-minor axis $\hat{b}$ to calculate the added mass which can be written as $m_{a}=\rho_{f} \pi \hat{b}^{2}$. Although the magnitude of $F_{a}$ is larger than that of $C_{T}$, the time-averaged value of $F_{a}$ is so marginal that it contributes little to the mean thrust, which was confirmed in $\mathrm{Bi}$ and $\mathrm{Zhu}(2019 \mathrm{~b})$ where a tethered swimming assumption was adopted as well. Under the tethered mode, the positive input of thrust sourced from added mass variation during the deflation phase is neutralized by the negative contribution during the inflation. In the subsequent study of the free-swimming scenario by Bi and Zhu (2019a), the added-mass related thrust was proven to account for a considerable portion of the mean thrust production.

The evolution of the Z-vorticity around the swimmer is presented in Fig. 7. It can be found that a pair of vortices, i.e., jet-induced vortices with clockwise and counterclockwise rotational directions, is shed from the mantle cavity. This is somewhat similar to the vorticity pattern observed from the flow around the live Lolliguncula brevis squid obtained by DPIV techniques in Bartol et al. (2008), especially the mode I pattern according to Bartol et al. (2009a). Interestingly, strong internal body vortices are also observed in this study, while they are much weaker in a previous study (Bi and Zhu, 2019b) where the Reynolds number is much smaller $(\operatorname{Re}=400)$. It is reasonable to conjecture that the Reynolds number affects the flow patterns around the system significantly, which will be discussed in the following section. A closer inspection of Fig. 7 reveals that the internal vortices and jet vortices are shed almost simultaneously at $t=2 / 3 T$. As demonstrated by their rotational directions, the internal vortices 
originate from the outer body surface, while the jet vortices originate from the inner body surface. Generally, the jet vortices, internal vortices and the wake external vortices attached on the outer surface are all symmetric concerning the horizontal midline within this period, although with opposite rotational directions above and below the axis of symmetry.

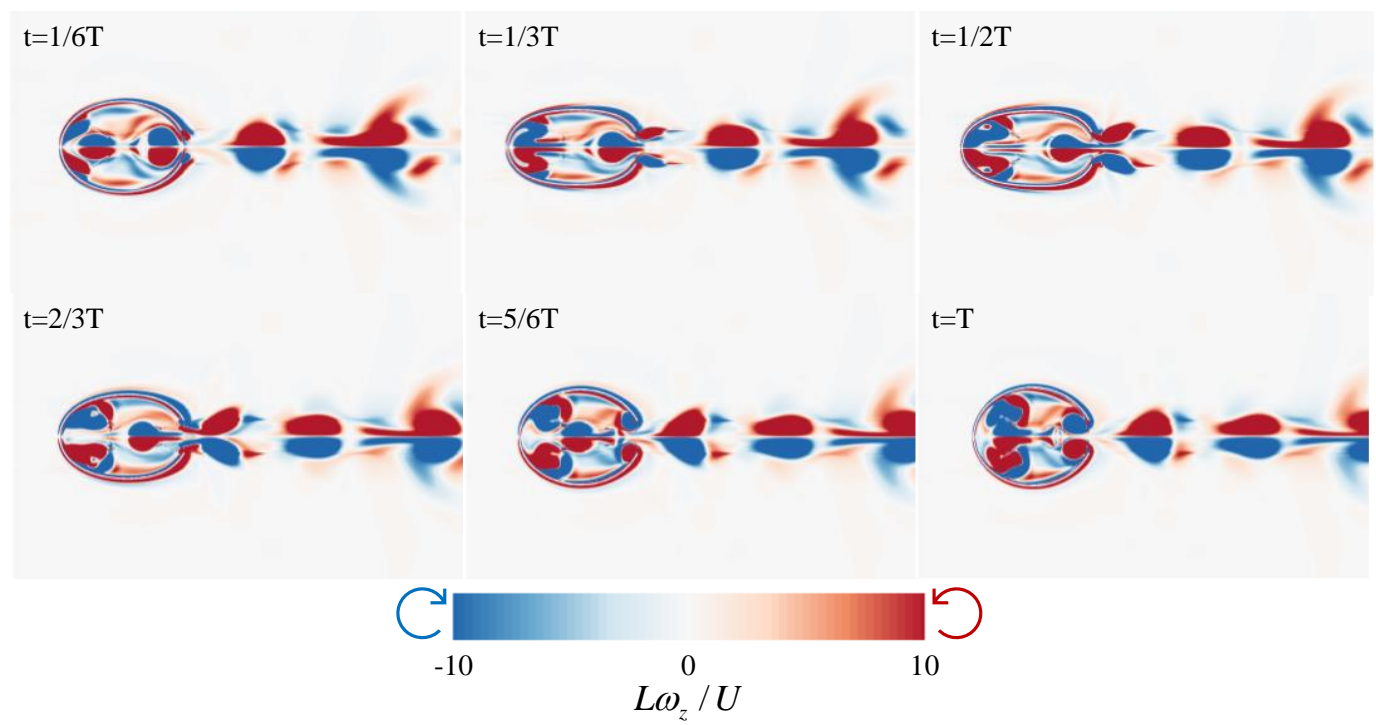

Fig. 7. The evolution of the Z-vorticity during one period.

In addition to the vorticity pattern, the propulsive thrust is also closely linked to the pressure distribution around the swimmer. Fig. 8 shows the evolution of the pressure distribution around the swimmer during one period. A comparison between Fig. 8 and Fig. 6(a) and (b) illustrates that the peak thrust at around 1/3 $T$ is directly related to the massive pressure difference around the body when it approaches the fully deflated state. Following the deflation, as the body is inflating, the upstream pressure starts to increase. Meanwhile, the downstream pressure decreases until $t=2 / 3 T$. As a result, during this process, the thrust diminishes continuously until the body experiences the maximum drag force near the instant at $t=2 / 3 T$. 


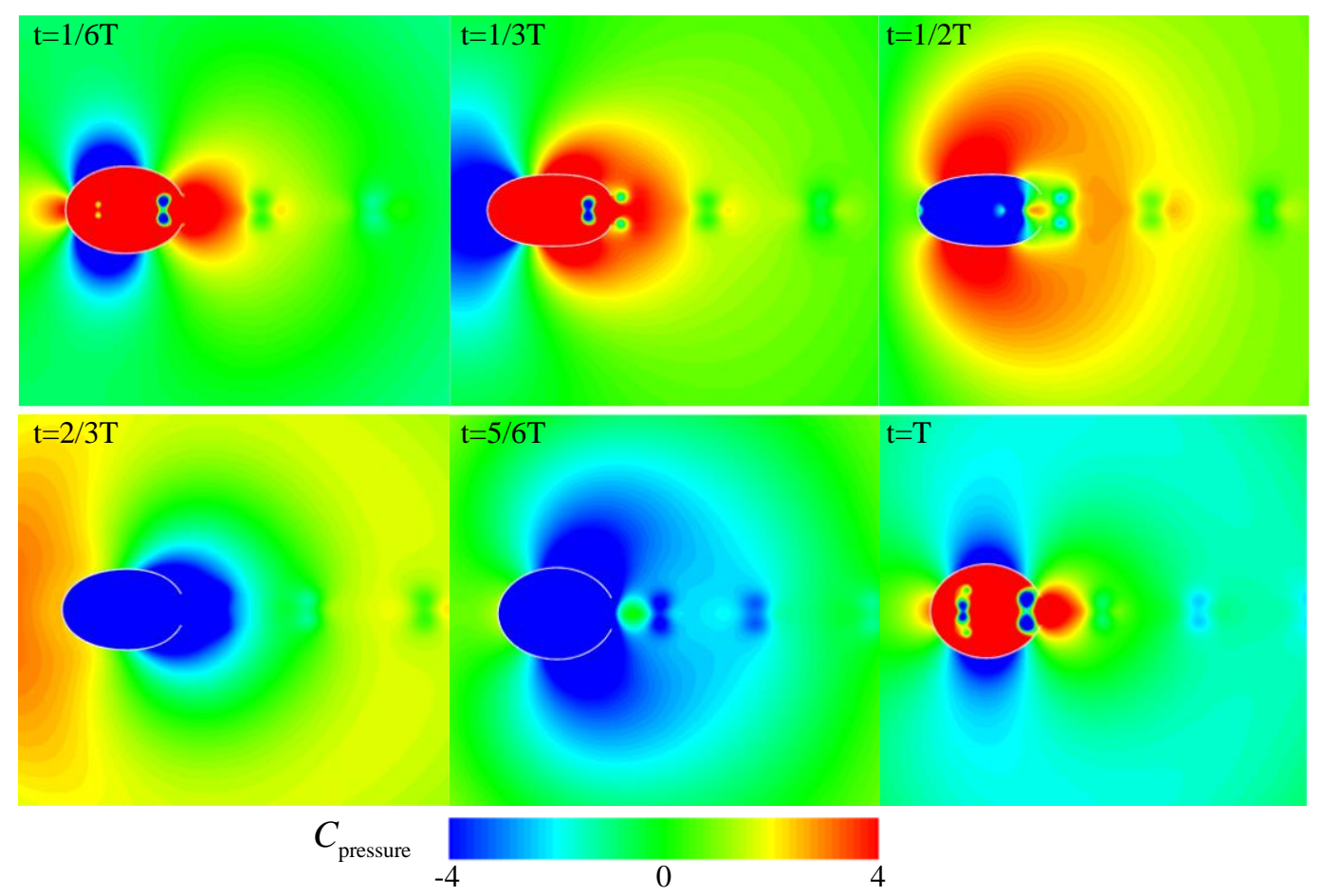

Fig. 8. The pressure contour during one period where $C_{\text {pressure }}=\left(p-p_{\infty}\right) / 0.5 \rho_{f} U^{2}$.

\subsection{Propulsion performance}

As can be observed in 4.1 , the vorticity pattern at $\mathrm{Re}=1 \times 10^{5}$ is considerably different from the one at lower Reynolds numbers (Bi and Zhu, 2019b). It is thus evident that fluid viscosity has a significant impact on the flow evolution around the system, and, consequently, the propulsive performance. Parametric studies are conducted here to explore the flow regimes when $\operatorname{Re}=50,400,3000$ and $1 \times 10^{5}$, which are within the range of Reynolds number squids experience throughout ontogeny (Bartol et al., 2008). Apart from the Reynolds number, the effects of $S t$ are also studied. Most of the non-dimensional parameters are kept the same as listed in Table I.

The time-averaged thrust coefficient $\overline{C_{T}}$, power expenditure coefficient $\overline{C_{P}}$, propulsion efficiency $\eta$ and the peak-to-peak displacement ratio of the co-vertex A (see Fig. 2) are shown in Fig. 9. In general, similar variation patterns of the four variables can be observed at $\mathrm{Re}=400$, 3000 and $1 \times 10^{5}$, while distinctive variation fashions are seen at $\mathrm{Re}=50$ where the viscous effect dominates. 
(a)

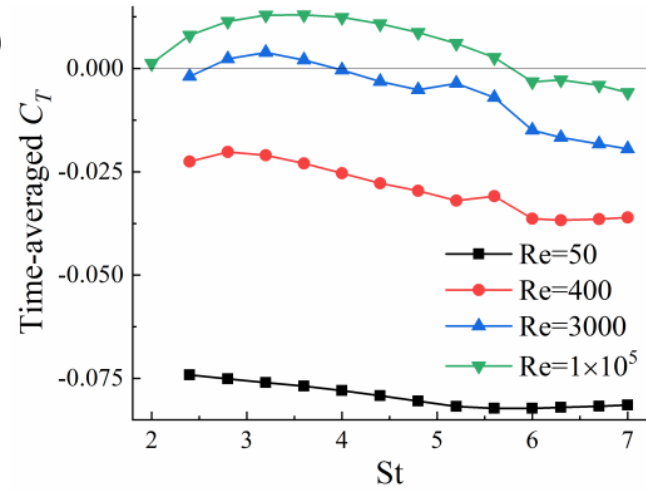

(c)

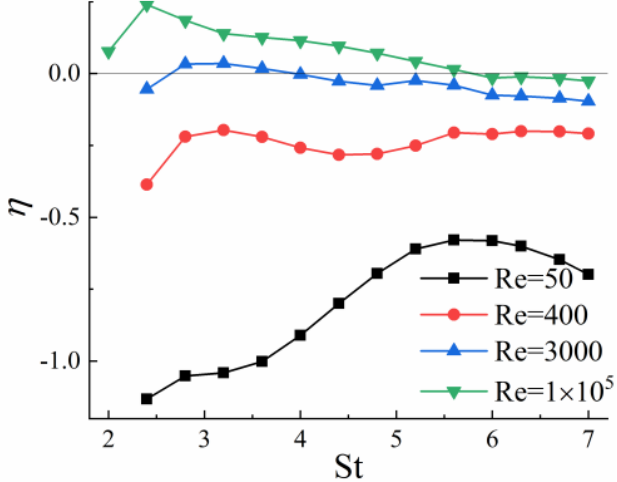

(b)

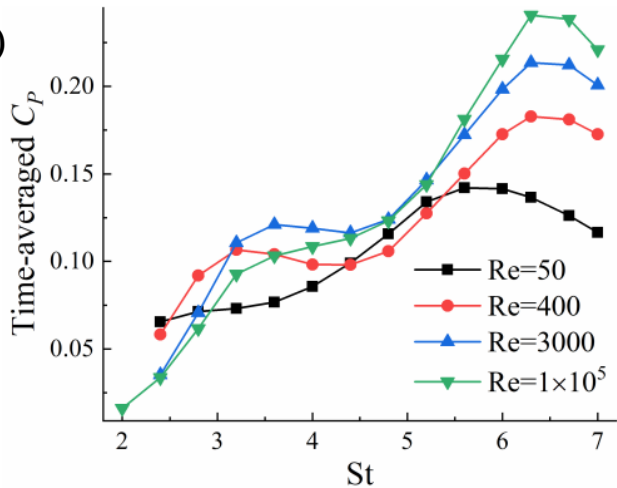

(d)

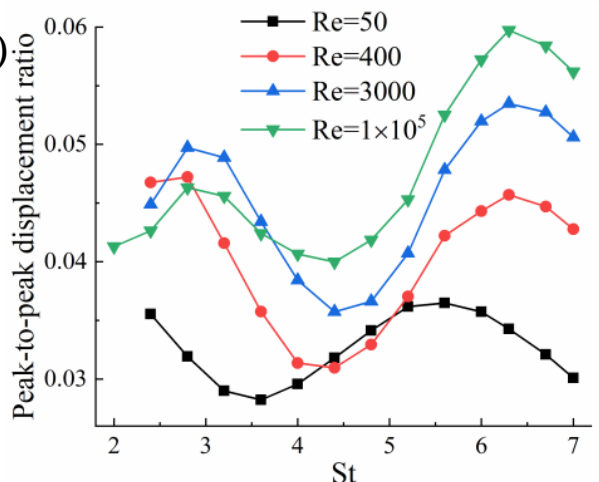

Fig. 9. The mean thrust (a), power expenditure coefficient (b), efficiency (c) and peak-to-peak displacement ratio of the co-vertex $A$ (d) as functions of $S t$ for different Reynolds number.

With the increase of the Reynolds number, the mean thrust increases at fixed actuation frequency $S t$ as shown in Fig. 9(a). When the flow is laminar, the swimmer does not produce mean thrust in most cases. Instead, it only experiences drag force, although this drag decreases at a higher Reynolds number. The time-averaged thrust peaks at $S t=3.2$ with a value of 0.0039 when $\mathrm{Re}=3000$, and at $S t=3.6$ with $\overline{C_{T}}=0.013$ when $\mathrm{Re}=1 \times 10^{5}$, yielding an increase by $200 \%$. Drag force is seen at low or high actuation frequencies in both cases. Similar variation patterns are seen in the efficiency plot in Fig. 9(c), where maximum efficiencies are observed at $S t=3.2$ and $S t=2.4$ for $\mathrm{Re}=3000$ and $1 \times 10^{5}$, respectively. Specifically, the optimal efficiency at $\operatorname{Re}=1 \times 10^{5}$ is $24.0 \%$, a significant increase by $586 \%$ from the optimal efficiency at $\mathrm{Re}=3000$ (around 3.5\%). As seen in Eq. (4), the power expenditure is directly associated with mantle displacement. As a result, their curves show some similarity, as depicted in Fig. 9(b) and (d). The maximum displacement of the co-vertex $A$ and maximum power expenditure are seen near the free vibration frequency $S t_{v}=6.3$, except for cases of $R e=50$ where the maximum is seen at a smaller St. With a closer inspection of Fig. 9(c) and (d), we also find a locally 
maximum displacement near $S t=2.8$ when $\mathrm{Re}$ is larger than 50. This second peak may correspond to another natural mode of the structure system, which has not been excited in our relaxation test in Appendix B.

The effect of the locomotion frequency has also been studied in Bi and Zhu (2019b) (see Fig. 11 in their work) at $\mathrm{Re}=400$. It would be interesting to compare it with the current results. The time-averaged thrust coefficients they predicted were in general larger than those in the present work by one order of magnitude. This is attributed to the fact that the deformation of the mantle body in their model was much more significant than what we achieve with the current activation mechanism. As a consequence, their model produced stronger jet flow, as mentioned in 4.1. Nevertheless, when higher Reynolds numbers are considered, the improvement in efficiency is dramatic according to our results. Namely, the maximum efficiency was below $15 \%$ in $\mathrm{Bi}$ and Zhu (2019b) at $\operatorname{Re}=400$, while our swimmer achieves an optimal efficiency of $25 \%$ at $\operatorname{Re}=1 \times 10^{5}$.

Resonant deformation of the mantle body may not be the best choice for the squid-like swimmer. For instance, while the globally maximum displacement is reached at the free vibration frequency $\left(S t_{\mathrm{v}}=6.3\right.$ ) when the Reynolds number is higher than 50, no time-mean thrust is generated there. Taking $\operatorname{Re}=1 \times 10^{5}$ as an example, the instantaneous thrust within one period at $S t=2,3.6$ and 6.3 are depicted in Fig. 10. At the free vibration frequency $S t=6.3$, the largest deformation of the mantle indeed yields a much larger peak thrust. However, the thrust production does not last long (less than 2/5 of the whole period). Although the magnitude of the maximum drag is smaller than that of the thrust, the resultant time-averaged thrust is still negative. In comparison, thrust production lasts more than half of the period at $S t=3.6$. As a result, the largest mean thrust is obtained at this actuation frequency.

The instantaneous thrust for different Reynolds numbers at $S t=3.2$ is compared against each other in Fig. 11. It can be found that in the laminar flow regime, the peak values of $C_{T}$ all increase with a higher Reynolds number. The maximum instantaneous peak values of $C_{T}$ among the four Reynolds numbers are seen at $\mathrm{Re}=3000$. Generally, a higher Reynolds number always results in an earlier occurrence of these maximum values. The variations of $V_{j}$ and 
$F_{a}$ at these four Reynolds numbers are similar to that of $C_{T}$ as shown in Fig. 11. Therefore, they are not shown here.

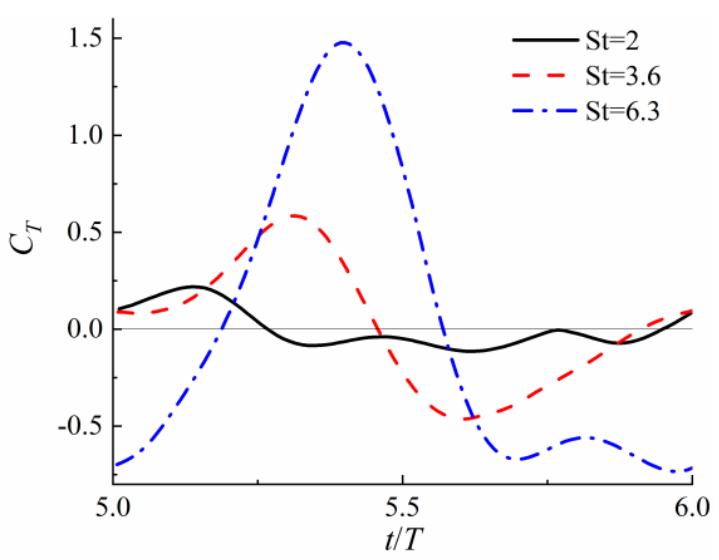

Fig. 10. The instantaneous thrust coefficient during one period at $\operatorname{Re}=1 \times 10^{5}$ for three St.

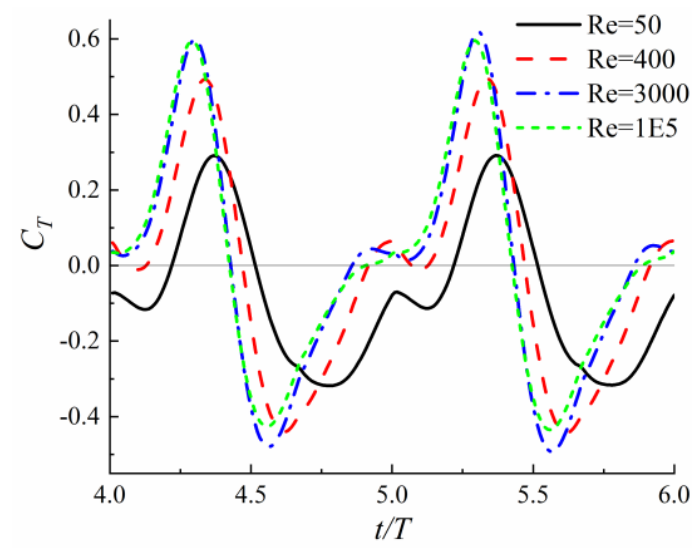

Fig. 11. The instantaneous thrust coefficient at $S t=3.2$ for different Re.

\subsection{Flow patterns and symmetry-breaking instability}

For interpretation of the propulsive performance under different parameters, visualization of the flow patterns is presented here. The Z-vorticity contours at the fully deflated state of the mantle at $S t=3.2$ for the four Reynolds numbers are presented in Fig. 12. With the increase of Re, the external body generated vortices are weaker, while the jet vortices become more dominant. At very low Re, i.e., $\mathrm{Re}=50$ and 400 , the jet vortices ejected from the intramantle and the internal body vortices dissipate quickly. Therefore, there are no noticeable vortex pairs in the wake or inside the mantle. Instead, the external body vortices, which are reminiscent of 
the vortices seen at the front surface of a circular cylinder in the laminar flow (Peppa and Triantafyllou, 2016), are the strongest, implying the drag force is dominating. This may explain that no thrust is produced for these two values of Re, as shown in Fig. 9(a). At $\operatorname{Re}=1 \times 10^{5}$, the jet vortices in the wake are the strongest, and the drag-force related external body vortices along the wall surface are highly suppressed, which contribute to the thrust generation directly.

(a)

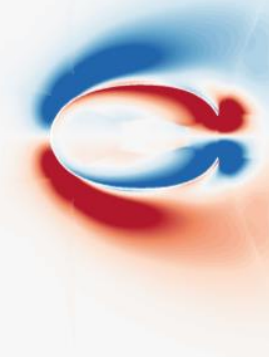

(c)

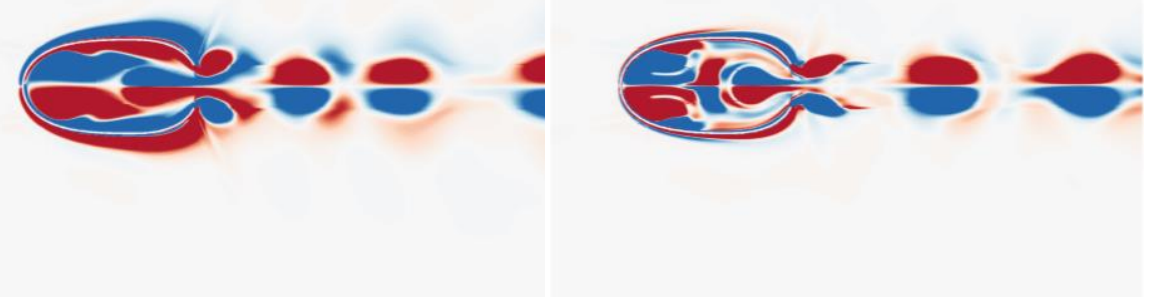

(b)

(d)

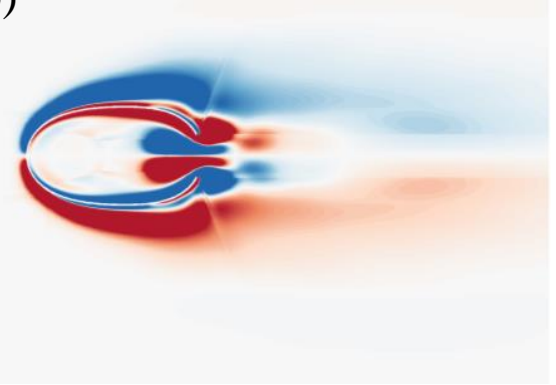

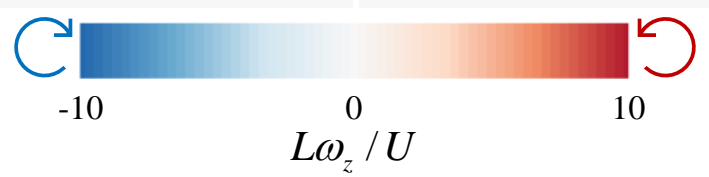

Fig. 12. The Z-vorticity contour at fully deflated state at $S t=3.2$ for different $R e=50$ (a), 400 (b), 3000 (c) and $1 \times 10^{5}$ (d).

In addition to fluid viscosity, the actuation frequency of the external force also plays a significant role in the flow pattern around the swimmer, as shown in Fig. 13. There are three vorticity patterns observed at different ranges of $S t$. At low frequency, e.g., $S t=2$, pattern I is presented where the external body and jet vortices are comparable in strength while internal body vortices are weak. This vorticity mode is associated with low thrust production. At pattern II, with $S t$ between 2.4 and 4.8, the jet vortices become dominant over the body-generated ones. Meanwhile, the internal body vortices grow stronger. Considerable thrust generation is seen within this pattern, e.g., $S t=3.6$ and 4.8, as shown in Fig. 9(a). High efficiencies are reached 
near the transition from pattern I to the jet vortices dominated pattern, i.e., at $S t=2.4$ and 2.8, as presented in Fig. 9(c). This is consistent with the findings in Bi and Zhu (2019b). In pattern III, with a further increase of $S t$, the drag-force related external body surface vortices become stronger again. In contrast, the jet vortices, the primary source of thrust production, fade in comparison with the body vortices. In consequence, there is no mean thrust generated in pattern III when the actuation frequency is high.

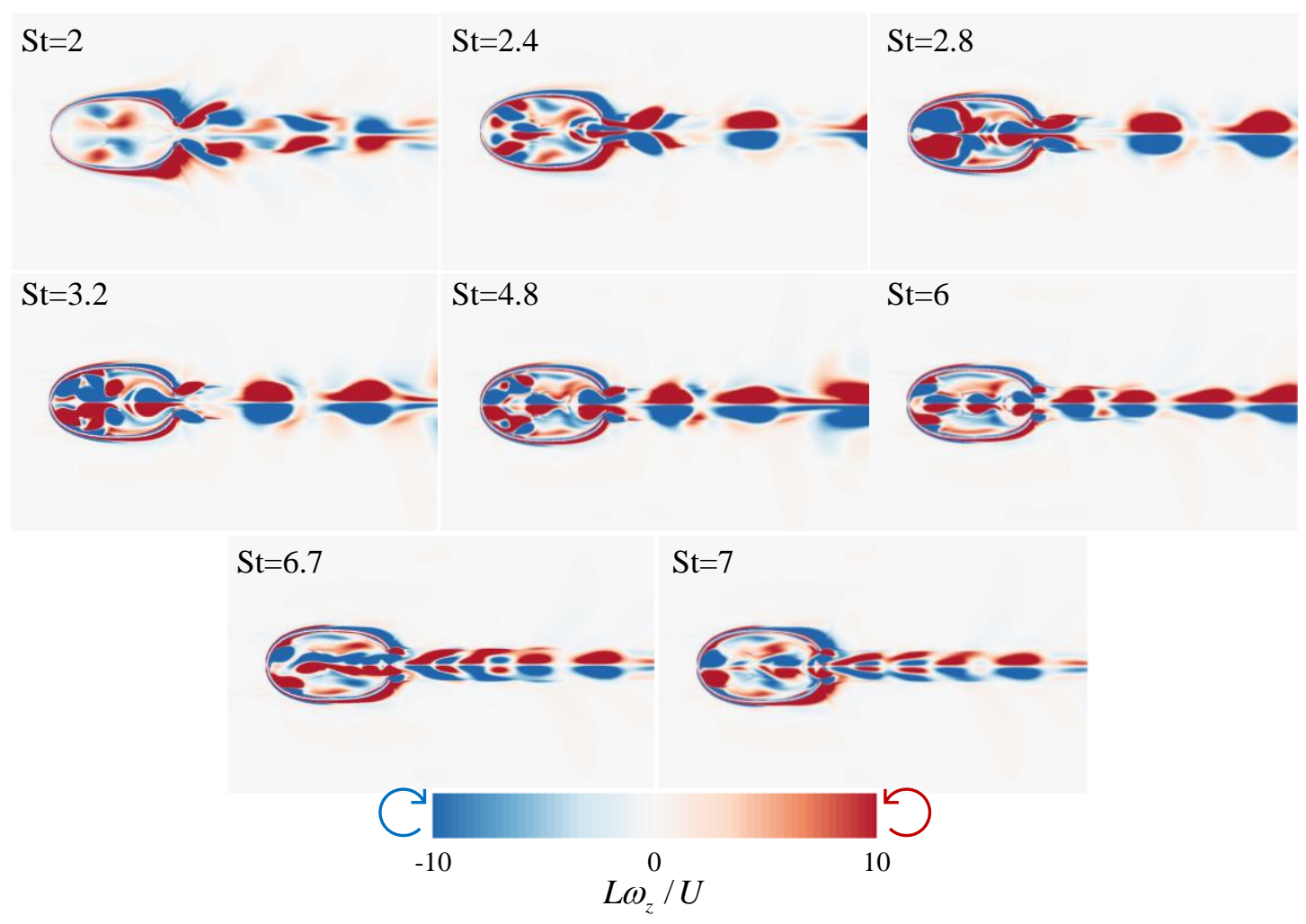

Fig. 13. The Z-vorticity contours around the swimmer at fully deflated state at $\mathrm{Re}=1 \times 10^{5}$.

With the proceeding of simulations, we find that the regular periodic deflation-inflation locomotion and resultant jet propulsion system are not durable after several cycles, especially at high Reynolds numbers. To demonstrate the evolution to irregular vibration and symmetry breaking, the time history of the Y position of the co-vertex $A$ and lift force $C_{y}$ in the $y$ direction are plotted in Fig. 14. The irregularity of the body deformation is noticeable after about eight cycles at $\operatorname{Re}=1 \times 10^{5}$, and one period later at $\operatorname{Re}=3000$. At lower $\operatorname{Re}$, the periodic vibration is maintained within all the ten periods. Another consequence of the asymmetric deformation is the production of lift force. As shown in Fig. 14(b), during regular periodic 
deflation-inflation locomotion, the lift force $C_{y}$ is negligible. However, the lift curves start to fluctuate from the seventh period for $\operatorname{Re}=3000$ and $1 \times 10^{5}$. The first peak lift of the former $\operatorname{Re}$ occurs slightly later than that of the latter Re. Generally, a higher Re leads to earlier appearance of the symmetry-break instability. This is reminiscent that squid hatchlings which operate at laminar flow $(\operatorname{Re}=1 \sim 100)$ employ continuous jet swimming more frequently than juveniles and adults which operate at higher Reynolds number $\left(\operatorname{Re}=10^{3} \sim 10^{6}\right)$ (Bartol et al., 2008). Burstand-coast swimming by these juveniles and adult squids may help them avoid this symmetrybreak instability in turbulent flow.

(a)

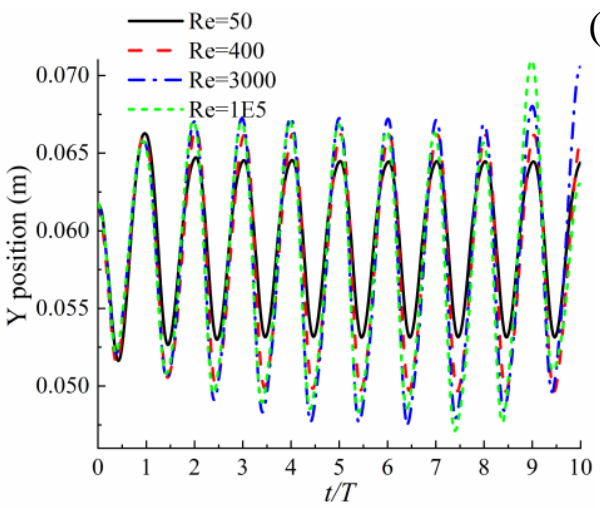

(b)

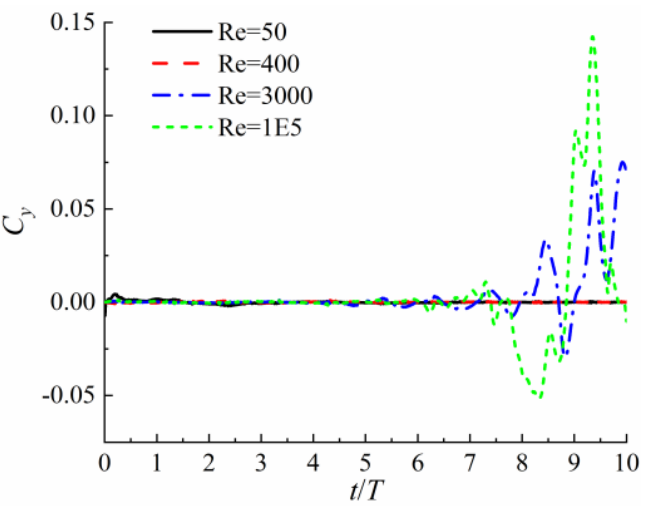

Fig. 14. The time history of the Y position of the co-vertex $A$ (a) and $C_{y}$ (b) at $S t=3.2$ for different Re.

The evolution of the Z-vorticity at $\operatorname{Re}=1 \times 10^{5}$ is presented in Fig. 15 to showcase the development of the symmetry-breaking instability (see also the supplementary material, video V1). The case at $\mathrm{Re}=400$ is also provided in Fig. 16 for a comparison. It can be found from Fig. 15 that before $t=6.60 T$, all the three types of vortices, i.e., the internal body vortices, the outer body surface vortices and jet vortices, are symmetric about the horizontal midline. From $t=7 T$ to $7.73 T$, the symmetry of the vortices inside the mantle body is broken, while the symmetry of the other vortex pairs generally remains. A comparison of Fig. 14 and Fig. 15 reveals that the irregularity of the body deformation and the lift force production are firstly sourced from unstructured internal body vortices. Afterwards, the symmetry breaking of the jet vortices is induced, which becomes noticeable after $t=8.60 \mathrm{~T}$. Similar wake symmetry breaking was reported in $\mathrm{Bi}$ and $\mathrm{Zhu}(2019 \mathrm{~b})$ at a much smaller Re of 400. In their study, the internal body 
vortices were not pronounced, and the deflation-inflation system instability was mainly due to the broken symmetry of the jet vortices in the wake. They expected that a smaller Reynolds number tended to delay the symmetry breaking. Indeed, our simulations indicate that the symmetry of the vortex pairs is well sustained at least for ten periods at $\mathrm{Re}=400$ as shown in Fig. 16. Under this scenario, the existing vortices dissipate quickly, and newly formed vortices avoid colliding with them to cause disturbance inside and outside the mantle body. To the knowledge of the authors, our results, for the first time, demonstrate the significant effect of internal body vortices on the system instability in jet propulsion at high Reynolds number condition.

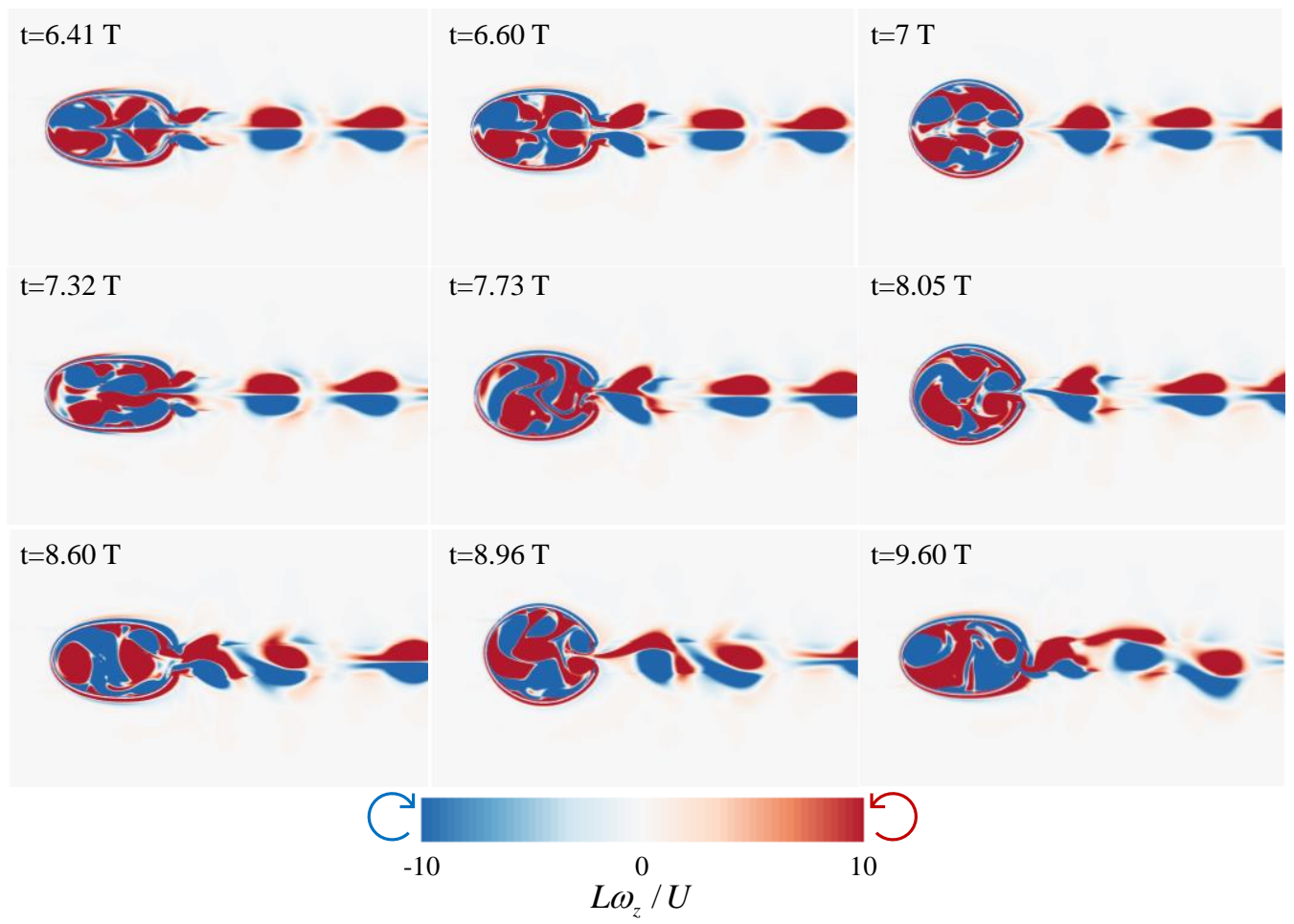

Fig. 15. The evolution of Z-vorticity contour around the swimmer at $\operatorname{Re}=1 \times 10^{5}$ and $S t=3.2$. 


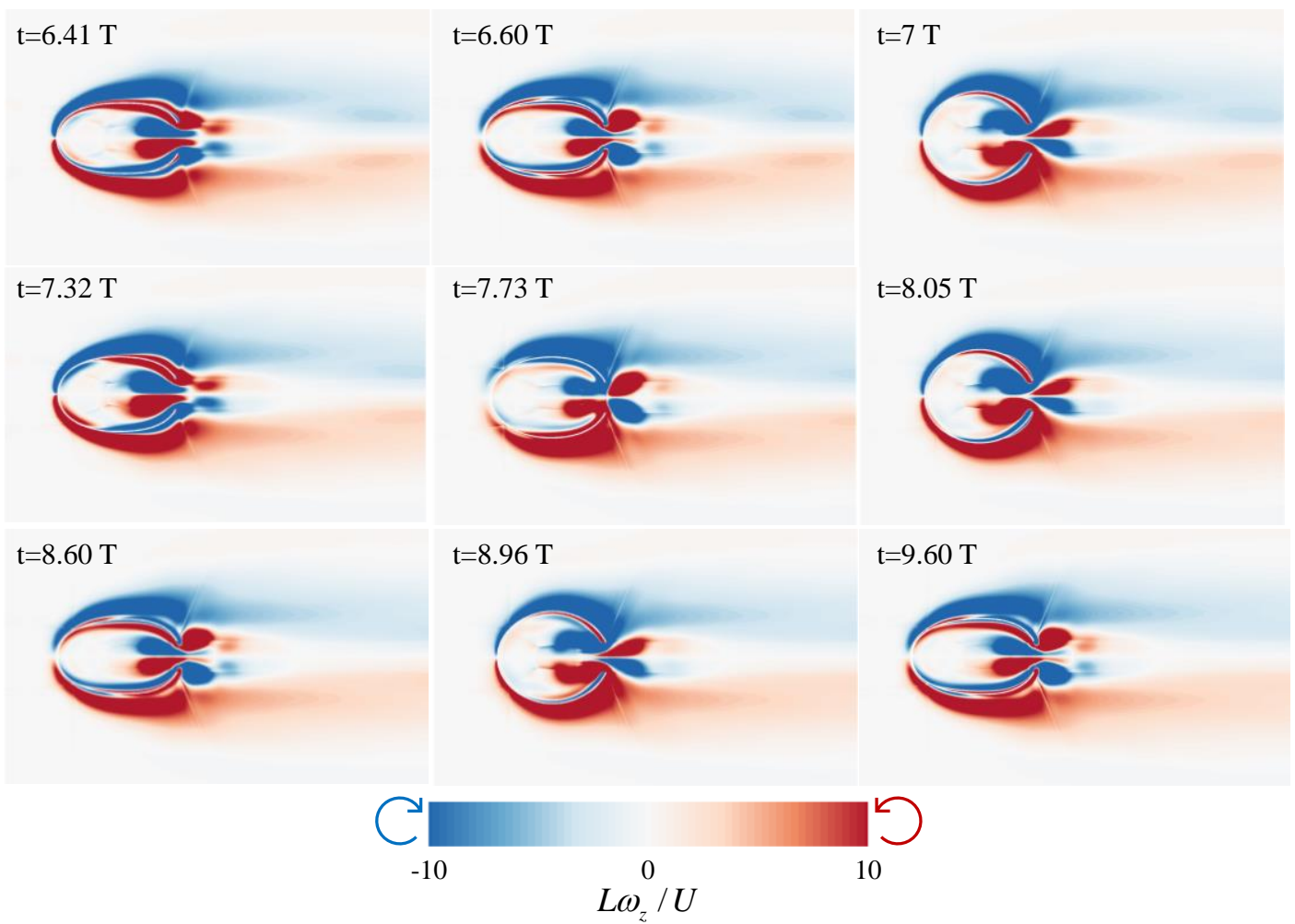

Fig. 16. The evolution of Z-vorticity contour around the swimmer at $\mathrm{Re}=400$ and $S t=3.2$.

\subsection{Effect of the nozzle size}

Jet flow, the main source of the thrust in jet propulsion, is directly influenced by the size of the nozzle which serves as the inlet and outlet. Here, two nozzle sizes, a large one with $R=0.14 L$ and a small one with $R=0.075 \mathrm{~L}$, are considered. It is noted that the free vibration frequencies used for the inflation phase are different for the two nozzle sizes. They are obtained from the free vibration study introduced in Appendix B. The parameters in the simulations for the large and small nozzle size are listed in Table II.

Table II. List of parameters used in the simulations for the two different nozzle sizes.

\begin{tabular}{cccccccc}
\hline $\operatorname{Re}$ & $K$ & $C_{\text {ef0 }}$ & $m^{*}$ & $a$ & $b$ & $R($ Large $)$ & $R($ Small $)$ \\
\hline $1 \times 10^{5}$ & 0.05 & 0.64 & 0.05 & $0.2 L$ & $0.15 L$ & $0.14 L$ & $0.075 L$ \\
\hline
\end{tabular}

The time-averaged thrust, power expenditure coefficient, efficiency, and the instantaneous jet speed at $S t=3.6$ are plotted in Fig. 17. It can be seen that under the parameters studied, at low 
actuation frequency, the mean thrust of the jetter with the small nozzle size is larger. With the increase of $S t$, its time-averaged thrust becomes lower than that for a large nozzle size. The peak mean thrust of the swimmer with the large nozzle is 0.012 at $S t=3.2$, an increase by $27 \%$ from the peak thrust (0.0094) of the swimmer with the small nozzle. The power expenditure of the two cases monotonously increases as the external force actuation frequency increases. The swimmer with the large nozzle size is more energy-saving compared to that with a small one. As a result of more thrust and less power expenditure, the swimmer with large nozzle is more efficient than the one with a small nozzle with the maximum efficiency difference $98 \%$ between them (with the efficiency of the latter as reference) at $S t=3.2$, as shown in Fig. 17(c). The variation patterns of $\overline{C_{T}}, \overline{C_{P}}$ and $\eta$ as a function of St suggest that the swimmer with a relatively large nozzle generally outperforms that with a small one in terms of propulsive capabilities.

(a)

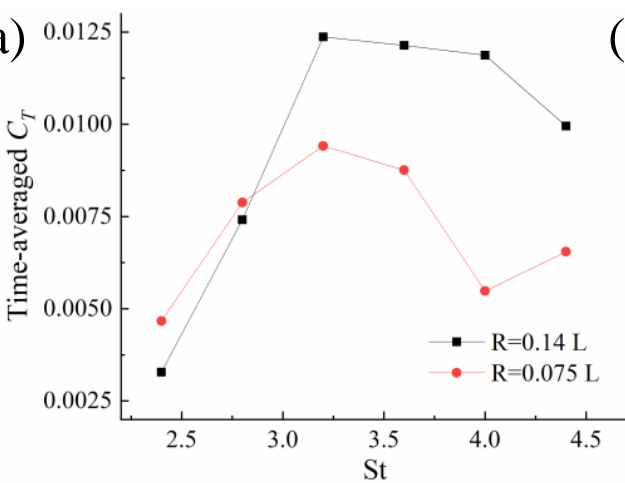

(c)

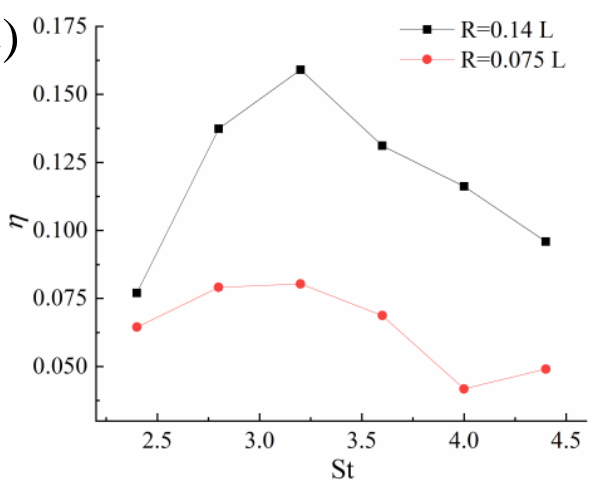

(b)

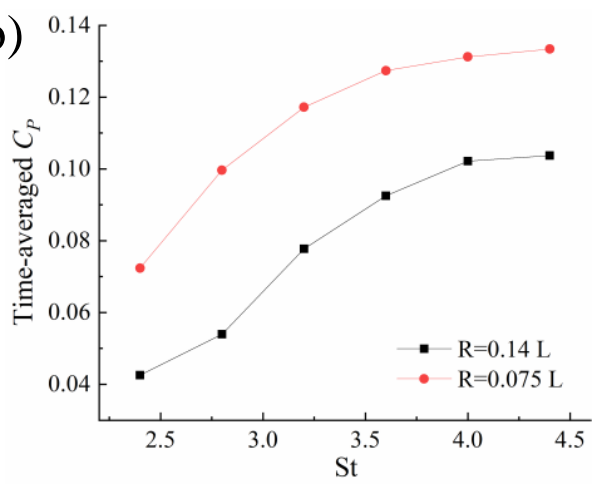

(d)

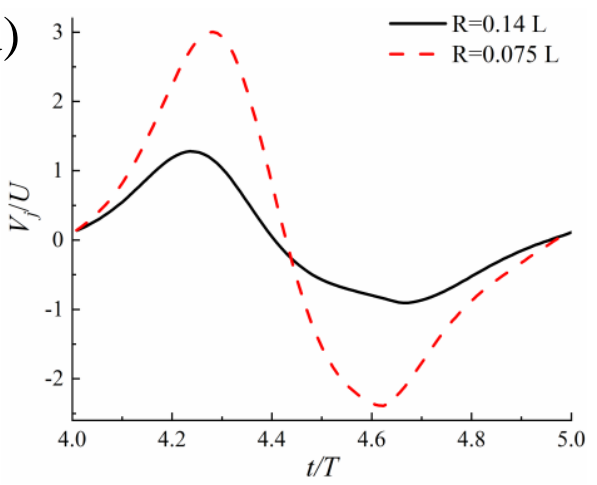

Fig. 17. The mean thrust (a), power expenditure coefficient (b), efficiency (c) and instantaneous jet speed within one period at $S t=3.6$ (d) of the squid-like jet swimmers with two different nozzle sizes. 
Although the time-averaged thrust of the system with the small nozzle is lower, its instantaneous maximum thrust coefficient $(0.61)$ is larger than that of the swimmer with the large nozzle (0.54). This implies that the swimmer with a small nozzle may achieve larger instantaneous burst speed. As expected, larger jet speed is also produced from the small nozzle as presented in Fig. 17(d). The large nozzle leads to the early emergence of peak jet velocity within a deflation-inflation locomotion period.

The vorticity pattern around the swimmers at different values of $S t$ for the two nozzle sizes are plotted in Fig. 18. At low frequency, e.g., $S t=2.4$, the internal body vortices and jet vortices are weak when the nozzle size is large. This is different from the small nozzle size case, whose vortices are much stronger. The main difference at this instant $(t=8 T)$ between the two vorticity patterns is that the symmetry of the vortex pairs around the swimmers with large nozzle size is still well sustained. In contrast, the other vorticity patterns present fully developed intramantle disturbance and anti-symmetrical jet vortex pairs.

In addition to the fluid viscosity, the nozzle size also has a significant influence on the development of irregularity of the locomotion system as the cycle continues, which is presented by the lift force production in Fig. 19. As can be seen, before the occurrence of remarkable asymmetry of vortices at $t=8 T$, the lift generation is noticeable from the sixth period. 


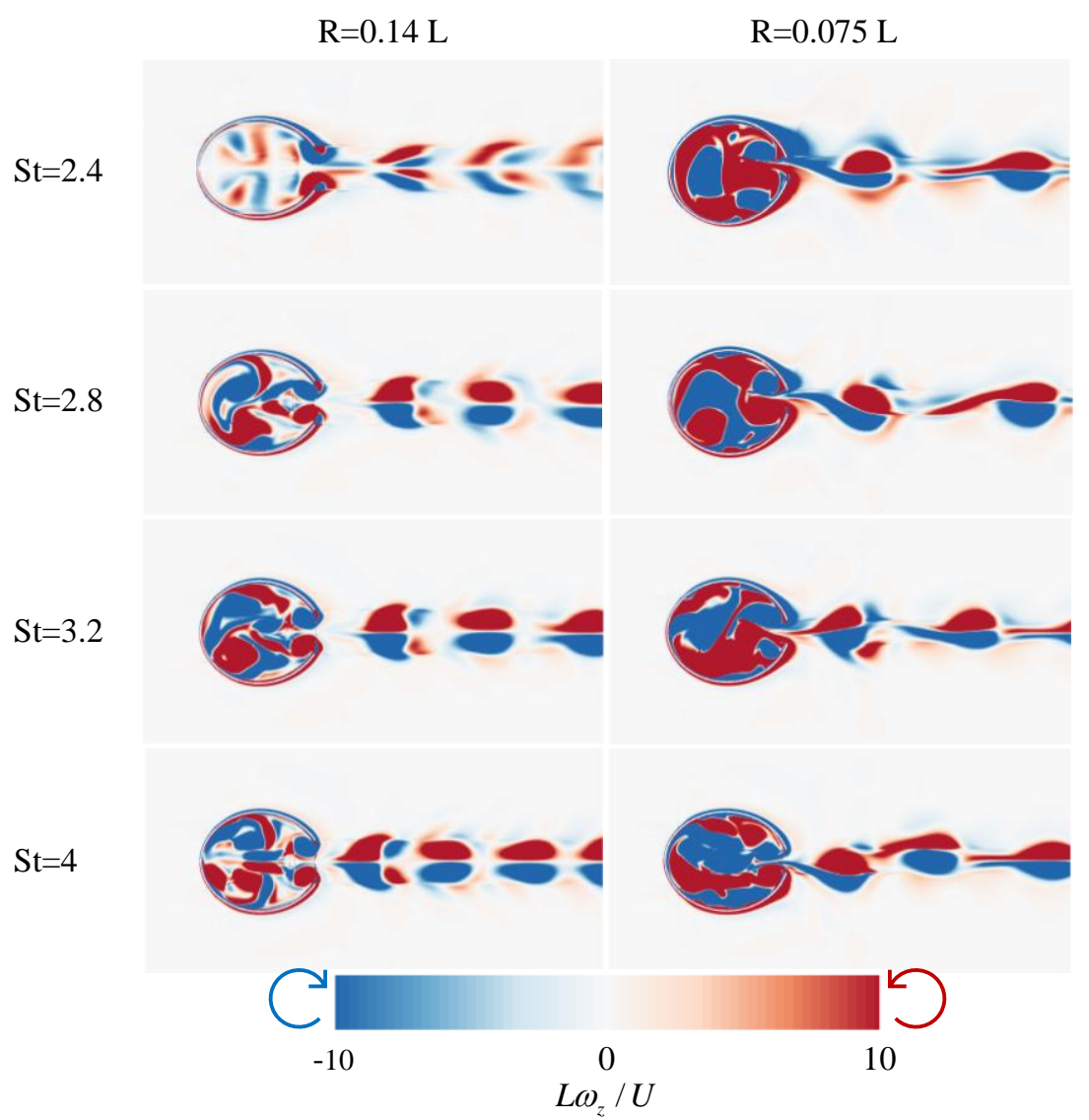

Fig. 18. Z-vorticity contour at $t=8 T$ for the two different nozzle sizes.

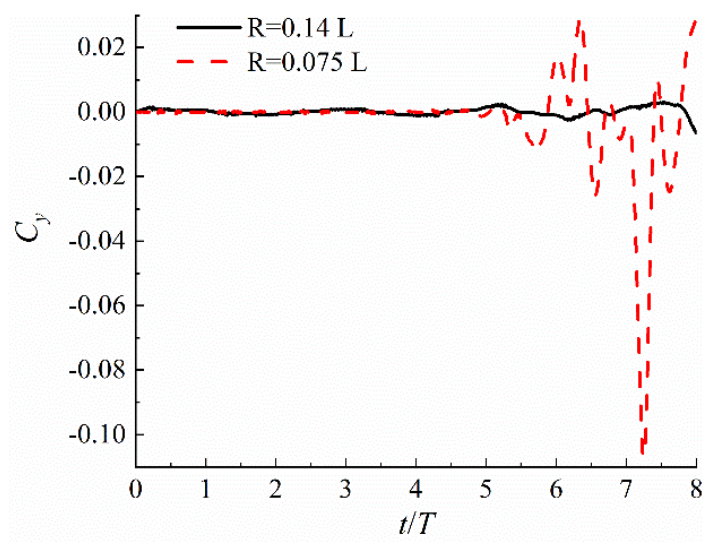

Fig. 19. The time history of $C_{y}$ at $S t=3.2$ for the two different nozzle sizes.

The evolution of the Z-vorticity contour around the swimmer with the small nozzle size is presented in Fig. 20 to demonstrate the development of the symmetry-breaking instability of the vorticity patterns. Similar to the phenomenon shown in Fig. 15, the symmetry breaking of the vortex pairs firstly happens inside the mantle body at around $t=5.98 T$. Afterwards, the 
internal body disturbance takes noticeable effect on the formation of jet vortices one period later, i.e., at about $t=7.67 T$. Regarding the vorticity pattern for the cases with the large nozzle sizes at $S t=3.2$, the wake symmetry is sustained at least until $t=8 T$, though the internal body vortices tend to become irregular at this instant as shown in Fig. 18. Consequently, the deformation of the body remains regular, and lift production is negligible until the end of the eighth period.

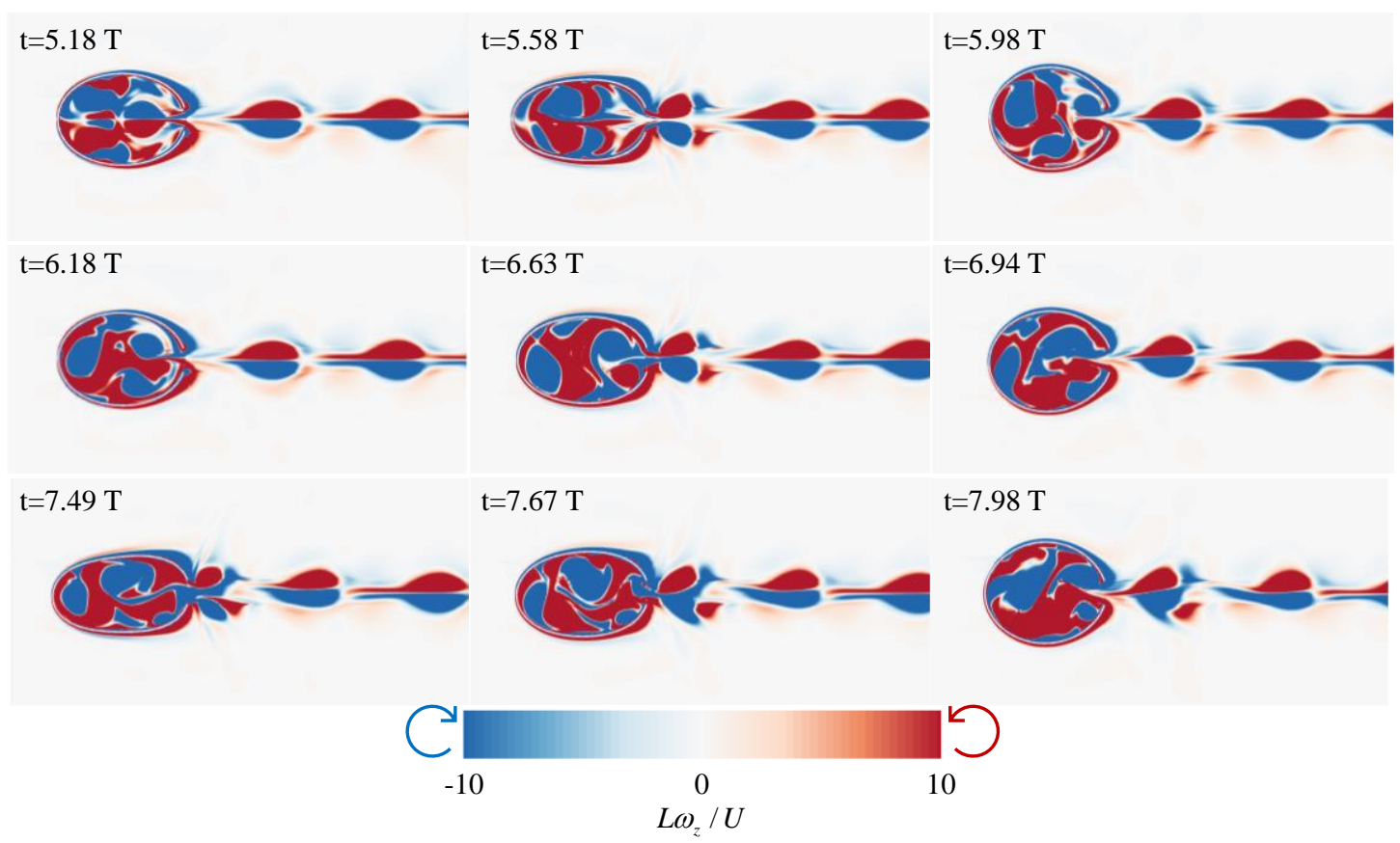

Fig. 20. The evolution of Z-vorticity contour at $S t=3.2$ around the squid-like simmer with the small nozzle size.

\section{Conclusions and discussion}

Inspired by the jet propulsion of squid and other cephalopods, a deflation-inflation propulsion system is proposed in this study. The two-dimensional squid-like swimmer is simplified as part of a hemi-ellipse with an open end which serves as the nozzle. External forcing is applied on the mantle body surface to actuate the deflation which mimics the constriction of the circular muscles of the squid. The mantle body shrinks under the actuation of the external force during the deflation phase. As a consequence, the fluid inside the chamber is ejected through the nozzle. After the external force is withdrawn, the mantle inflates as a result of the release of the elastic energy in the flexible body structure. During this inflation phase, the fluid enters the 
chamber through the nozzle, which will then be expelled in the next ejection. Our simulation results indicate that periodic deflation-inflation locomotion and thrust production (see Fig. 5 and Fig. 6) are achieved under this mechanism.

The effect of Reynolds number on the jet propulsion is investigated systematically. Our results show that a higher Reynolds number yields larger mean thrust generation under the parameters studied. This thrust augmentation may be attributed to the suppressed body-generated vortices near the outer body surface, which is an indicator of drag force. Additionally, the resonant frequency of the mantle structure which was excluded in Bi and Zhu (2019b) is determined, and results indicate that although maximum vibration displacement is observed near the resonant frequency, it does not yield better propulsion performance. Peak time-averaged thrust is observed as the actuation frequency is varied for all the four Reynolds numbers. The optimal efficiency is presented at a relatively low external force actuation frequency $S t$ (see Fig. 9(c)). Within the range of parameters considered in this study, the peak efficiency reaches $25 \%$, much higher than the one in Bi and Zhu (2019b) (15\%). The visualization of flow patterns shows that three modes can be observed with the increase of St. In pattern I, the body-generated vortices are comparable in strength with the jet vortices. Pattern II is dominated by jet vortices, and the body-shed vortices become more dominating in pattern III. The most efficient jet propulsion is seen near the transition from pattern I to pattern II when jet vortices start to dominate, which is consistent with the findings in Bi and Zhu (2019b).

In Bi and Zhu (2019b), the symmetry-breaking instability at $\mathrm{Re}=400$ was demonstrated after the inflation-deflation locomotion proceeds several cycles. They expected that a higher Reynolds number tends to enhance instability. Indeed, our model shows that the higher the Reynolds number is, the earlier the irregular deformation and vortices patterns occur (see Fig. 14 and Fig. 15). However, their numerical model only emphasized the importance of wake instability. Instead, our simulation results indicate that the mechanism of the system instability seen at low Reynolds number (laminar flow) may not apply to the turbulent flow under high Re scenarios. Specially, under laminar flow scenarios, the asymmetry is mainly seen in the wake jet vortex pairs, while the effect of internal vortices remains insignificant. In comparison, at a much higher Reynolds number in this study, the disturbance occurs firstly in the intramantle 
vortices whose symmetry is broken during the collision between newly formed and vortices formed earlier. Around one cycle later, this disturbance is reflected in the wake jet vortices noticeably.

Despite the fact that the current study is strictly two dimensional, the symmetry-breaking phenomenon may still have implications in the swimming of real squid, in which burst-andcoast jet along with the fin oscillation is employed. At low Re conditions where fluid viscosity is prominent, continuous swimming is more advantageous than burst-and-coast swimming (Weihs, 1974). Therefore, more frequent contractions with less coasting were predicted to be favoured by squid hatchlings swimming in the laminar flow environment by Bartol et al. (2008), which was in line with biological measurement (Thompson and Kier, 2001). In comparison, fin activations are more frequently utilized by juvenile and adults squids at higher Reynolds numbers. Based on our results, it is sensible to conjecture that the intermittent burst-and-coast swimming not only improves the efficiency but also suppress the symmetry-breaking instability of the internal body vortices. As indicated by Bi and Zhu (2019b), some specific measures may be needed to mitigate the effect of this instability during the design of jet-propulsion inspired underwater vehicle or propulsors, e.g., via active control of the body deformation to change the evolution of the internal vortices pattern.

For the sake of completeness, the effect of nozzle size on the propulsion performance is explored as well. Our results indicate that although a narrow nozzle may increase the instantaneous thrust production, it does not always benefit the time-averaged thrust or efficiency. Besides, a small nozzle size leads to stronger vortices which are more likely to induce symmetry-breaking instability (see Fig. 19 and Fig. 20).

Finally, our current study focuses on a two-dimensional swimmer which bears some resemblance to the jet propulsion of squid. Although turbulent flow is considered, which renders our model closer to the practical scenarios of real squid swimming, the exploration of the significant three-dimensional nozzle-shed vortex rings is not possible for a two-dimensional model. For example, it is known that the formation and evolution of the jet flow and vortices are closely correlated with a dimensionless parameter called formation number, which is not considered in this work. Three-dimensional simulations are needed to understand the 
underlying mechanism of the jet modes observed from live swimming squids in (Bartol et al., 2008).

\section{Supplementary material}

See supplementary material for the video showing the development of the symmetry-breaking at $\operatorname{Re}=1 \times 10^{5}, S t=3.2$ and $R=0.1 \mathrm{~L}$.

\section{Acknowledgement}

This research is supported by an EPSRC Supergen ORE Hub Flexible Fund Program Grant “Autonomous Biomimetic Robot-fish for Offshore Wind Farm Inspection" (EP/S000747/1). This work used the Cirrus UK National Tier-2 HPC Service at EPCC (http://www.cirrus.ac.uk) funded by the University of Edinburgh and EPSRC (EP/P020267/1) and ARCHIE-WeSt High Performance Computer (www.archie-west.ac.uk) based at the University of Strathclyde. The first author thanks China Scholarship Council (CSC) for financial support during his study in the UK.

\section{Data availability}

The data that support the findings of this study are openly available at the following URL/DOI: https://doi.org/10.15129/6007462b-af7b-465a-b4db-70be63610e3a.

\section{Appendix A. Validation}

This case serves as a validation of the $k-\omega$ turbulence model in the in-house fluid solver by simulating the two-dimensional flow over a NACA0012 airfoil. The Reynolds number for this simulation is $\operatorname{Re}=U c / v=3 \times 10^{6}$, where $c$ is the chord length of the airfoil, which is identical to the experimental data in (Abbott and Von Doenhoff, 2012). The height of the first cell is $1.0 \times 10^{-5} c$, corresponding to the value of $y^{+}$approximately 0.2 , which is smaller than 1 to sufficiently resolve the flow in the inner boundary layer. The uniform flow over the airfoil with the angle of $0^{\circ}, 3^{\circ}, 5^{\circ}, 9^{\circ}$ and $12^{\circ}$ when $\operatorname{Re}=3 \times 10^{\circ}$ is simulated. The results are compared with the previous numerical (Eleni et al., 2012) and experimental (Abbott and Von 
Doenhoff, 2012) data in Fig. 21. As seen in Fig. 21(b), the lift coefficients by the present simulations are slightly higher than the others as the angle of attack increases, but the discrepancy is still acceptable. The drag coefficients by numerical simulations are all slightly higher than the experimental results in (Abbott and Von Doenhoff, 2012). In general, the present results have good agreement with that in previous studies.
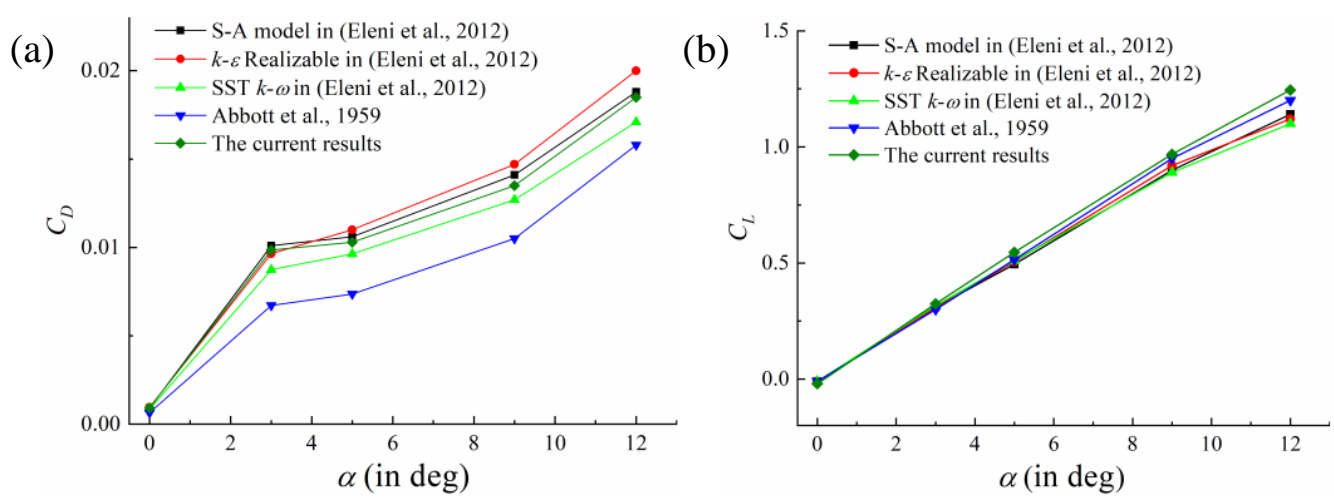

Fig. 21. Comparisons between experimental data from (Abbott and Von Doenhoff, 2012) and three different turbulent models simulation results in Eleni et al. (2012) of the drag $C_{D}$ (a) and lift $C_{L}$ (b) coefficient.

\section{Appendix B. Free vibration study}

To find the free vibration frequency of the structure, we performed a numerical relaxation study where a constant external force with $C_{\text {efo }}=0.64$, i.e., $F_{\text {ef }}(t)=0.5 \rho_{f} U_{\infty}^{2} L \cdot 0.64$, is applied on the mantle surface and then unloaded to let the body oscillate freely in the fluid. The response of the co-vertex $A$ of the elliptical body shown in Fig. 2 is recorded and presented in Fig. 22, at $K=0.05$ under different Reynolds number, and at $\mathrm{Re}=1 \times 10^{5}$ as stiffness is varied, respectively. It can be found from Fig. 22(a) that the vibration amplitude of the body decays faster as the fluid viscosity is larger (smaller Re). The damping effect is pronounced at $\mathrm{Re}=50$ where the vibration fades out much quicker than the others, which have also been demonstrated in (Hoover and Miller, 2015). Nevertheless, the varied Reynolds number does not change the vibration frequency much, as the difference among them are within $2 \%$ with the value at $\operatorname{Re}=1 \times 10^{5}$ as a reference, i.e., $S t_{\mathrm{v}}=f_{\text {vibration }} L / U=6.3$. Another significant factor affecting 
the vibration response of the mantle cavity is its structural stiffness, as shown in Fig. 22(b). As expected, the stiffer structure results in a higher-frequency oscillation and when the stiffness is sufficiently large, the response curves approach to each other, e.g., at $K=0.05$ and $K=0.06$. It can be found that deflation and inflation last for almost the equal period of time within one vibration cycle. Therefore, the passive inflation period $T_{\mathrm{i}}$ is chosen as half of the free vibration period in this study.
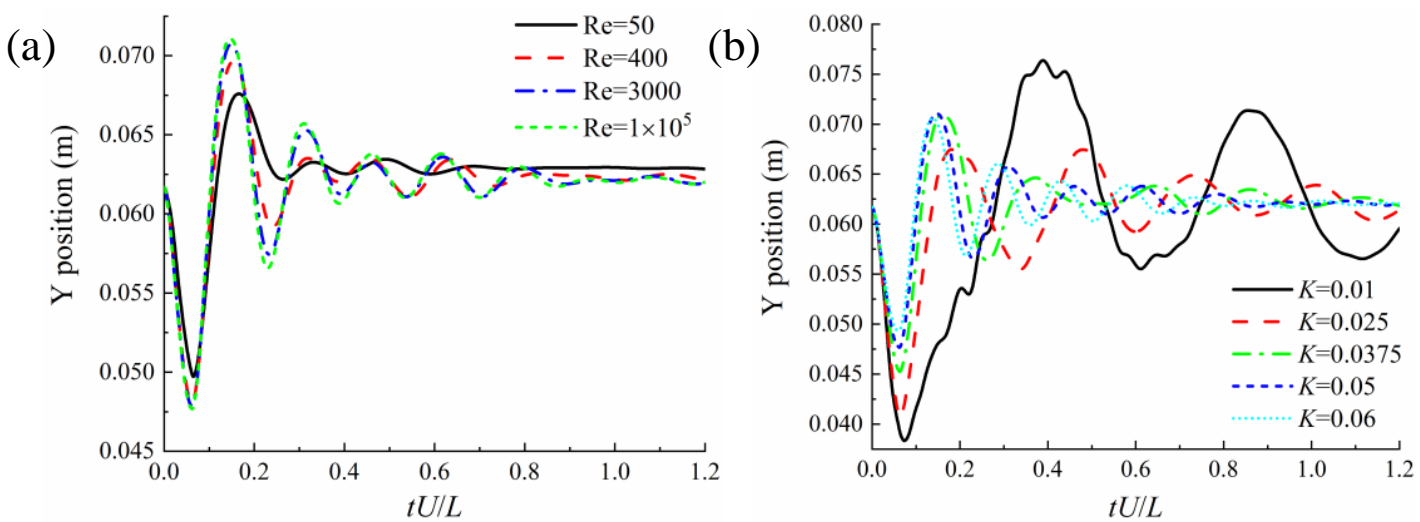

Fig. 22. The oscillation of the $Y$ position of the co-vertex $A$ as a function of non-dimensional time when $K=0.05$ at different Re number (a), and when $\operatorname{Re}=1 \times 10^{5}$ at different stiffnesses (b).

\section{References}

Abbott, I.H., Von Doenhoff, A.E., 2012. Theory of wing sections, including a summary of airfoil data. Courier Corporation.

Anderson, E.J., Grosenbaugh, M.A., 2005. Jet flow in steadily swimming adult squid. Journal of Experimental Biology 208, 1125-1146.

Bartol, I.K., Krueger, P.S., Jastrebsky, R.A., Williams, S., Thompson, J.T., 2016. Volumetric flow imaging reveals the importance of vortex ring formation in squid swimming tail-first and arms-first. Journal of Experimental Biology 219, 392-403.

Bartol, I.K., Krueger, P.S., Stewart, W.J., Thompson, J.T., 2009a. Hydrodynamics of pulsed jetting in juvenile and adult brief squid Lolliguncula brevis: evidence of multiple jetmodes' and their implications for propulsive efficiency. Journal of Experimental Biology 212, 1889-1903. Bartol, I.K., Krueger, P.S., Stewart, W.J., Thompson, J.T., 2009b. Pulsed jet dynamics of squid hatchlings at intermediate Reynolds numbers. Journal of Experimental Biology 212, 1506-1518. Bartol, I.K., Krueger, P.S., Thompson, J.T., Stewart, W.J., 2008. Swimming dynamics and propulsive efficiency of squids throughout ontogeny. Integrative and Comparative Biology 48, 720-733.

Bi, X., Zhu, Q., 2018. Numerical investigation of cephalopod-inspired locomotion with intermittent bursts. Bioinspiration \& biomimetics 13, 056005. 
Bi, X., Zhu, Q., 2019a. Dynamics of a squid-inspired swimmer in free swimming. Bioinspiration \& Biomimetics 15, 016005.

Bi, X., Zhu, Q., 2019b. Fluid-structure investigation of a squid-inspired swimmer. Physics of Fluids 31, 101901.

Bi, X., Zhu, Q., 2020. Pulsed-jet propulsion via shape deformation of an axisymmetric swimmer. Physics of Fluids 32, 081902.

Bungartz, H.-J., Lindner, F., Gatzhammer, B., Mehl, M., Scheufele, K., Shukaev, A., Uekermann, B., 2016. preCICE-a fully parallel library for multi-physics surface coupling. Comput Fluids 141, 250258.

Christianson, C., Cui, Y., Ishida, M., Bi, X., Zhu, Q., Pawlak, G., Tolley, M., 2020. Cephalopodinspired robot capable of cyclic jet propulsion through shape change. Bioinspiration \& Biomimetics, In press.

Costello, J.H., Colin, S.P., Dabiri, J.O., Gemmell, B.J., Lucas, K.N., Sutherland, K.R., 2020. The Hydrodynamics of Jellyfish Swimming. Annual Review of Marine Science 13.

Dhondt, G., 2004. The finite element method for three-dimensional thermomechanical applications. John Wiley \& Sons.

Eleni, D.C., Athanasios, T.I., Dionissios, M.P., 2012. Evaluation of the turbulence models for the simulation of the flow over a National Advisory Committee for Aeronautics (NACA) 0012 airfoil. Journal of Mechanical Engineering Research 4, 100-111.

Gosline, J.M., DeMont, M.E., 1985. Jet-propelled swimming in squids. Sci Am 252, 96-103.

Gosline, J.M., Steeves, J.D., Harman, A.D., Demont, M.E., 1983. Patterns of circular and radial mantle muscle activity in respiration and jetting of the squid Loligo opalescens. Journal of Experimental Biology 104, 97-109.

Han, P., Lauder, G.V., Dong, H., 2020. Hydrodynamics of median-fin interactions in fish-like locomotion: Effects of fin shape and movement. Physics of Fluids 32, 011902.

Hoover, A., Miller, L., 2015. A numerical study of the benefits of driving jellyfish bells at their natural frequency. Journal of theoretical biology 374, 13-25.

Hoover, A.P., Griffith, B.E., Miller, L.A., 2017. Quantifying performance in the medusan mechanospace with an actively swimming three-dimensional jellyfish model. J Fluid Mech 813, 1112 1155 .

Hou, T., Yang, X., Su, H., Jiang, B., Chen, L., Wang, T., Liang, J., 2019. Design and Experiments of a Squid-Like Aquatic-Aerial Vehicle with Soft Morphing Fins and Arms, 2019 International Conference on Robotics and Automation (ICRA). IEEE, pp. 4681-4687.

Jameson, A., Schmidt, W., Turkel, E.L.I., 1981. Numerical solution of the Euler equations by finite volume methods using Runge Kutta time stepping schemes, 14th Fluid and Plasma Dynamics Conference. American Institute of Aeronautics and Astronautics.

Lauder, G., Madden, P., Tangorra, J., Anderson, E., Baker, T., 2011. Bioinspiration from fish for smart material design and function. Smart Materials and Structures 20, 094014.

Liu, W., Xiao, Q., Zhu, Q., 2016. Passive Flexibility Effect on Oscillating Foil Energy Harvester. AIAA Journal 54, 1172-1187.

Luo, Y., Xiao, Q., Shi, G., Pan, G., Chen, D., 2020a. The effect of variable stiffness of tuna-like fish body and fin on swimming performance. Bioinspiration $\&$ Biomimetics, In press. 
Luo, Y., Xiao, Q., Shi, G., Wen, L., Chen, D., Pan, G., 2020b. A fluid-structure interaction solver for the study on a passively deformed fish fin with non-uniformly distributed stiffness. Journal of Fluids and Structures 92, 102778.

Megill, W.M., Gosline, J.M., Blake, R.W., 2005. The modulus of elasticity of fibrillin-containing elastic fibres in the mesoglea of the hydromedusa Polyorchis penicillatus. Journal of Experimental Biology 208, 3819-3834.

Mehl, M., Uekermann, B., Bijl, H., Blom, D., Gatzhammer, B., van Zuijlen, A., 2016. Parallel coupling numerics for partitioned fluid-structure interaction simulations. Computers \& Mathematics with Applications 71, 869-891.

Miles, J.G., Battista, N.A., 2019. Naut your everyday jellyfish model: Exploring how tentacles and oral arms impact locomotion. Fluids 4, 169.

O’Dor, R.K., 2013. How squid swim and fly. Canadian journal of zoology 91, 413-419.

Park, S.G., Kim, B., Lee, J., Huang, W.-X., Sung, H.J., 2015. Dynamics of prolate jellyfish with a jetbased locomotion. Journal of Fluids and Structures 57, 331-343.

Peppa, S., Triantafyllou, G.S., 2016. Sensitivity of two-dimensional flow past transversely oscillating cylinder to streamwise cylinder oscillations. Physics of Fluids 28, 037102.

Renda, F., Giorgio-Serchi, F., Boyer, F., Laschi, C., 2015. Modelling cephalopod-inspired pulsed-jet locomotion for underwater soft robots. Bioinspiration \& biomimetics 10, 055005.

Sadeghi, M., 2004. Parallel computation of three-dimensional aeroelastic fluid-structure interaction. University of California, Irvine.

Sadeghi, M., Yang, S., Liu, F., Tsai, H., 2003. Parallel computation of wing flutter with a coupled Navier-Stokes/CSD method, 41st Aerospace Sciences Meeting and Exhibit, p. 1347.

Salazar, R., Fuentes, V., Abdelkefi, A., 2018. Classification of biological and bioinspired aquatic systems: A review. Ocean Engineering 148, 75-114.

Shi, G., Xiao, Q., Zhu, Q., Liao, W., 2019. Fluid-structure interaction modeling on a 3D raystrengthened caudal fin. Bioinspiration \& biomimetics 14, 036012.

Spagnolie, S.E., Shelley, M.J., 2009. Shape-changing bodies in fluid: hovering, ratcheting, and bursting. Physics of Fluids 21, 013103.

Steele, S.C., Weymouth, G.D., Triantafyllou, M.S., 2017. Added mass energy recovery of octopusinspired shape change. J Fluid Mech 810, 155-174.

Thompson, J.T., Kier, W.M., 2001. Ontogenetic changes in mantle kinematics during escape-jet locomotion in the oval squid, Sepioteuthis lessoniana Lesson, 1830. The Biological Bulletin 201, 154166.

Thompson, J.T., Kier, W.M., 2006. Ontogeny of mantle musculature and implications for jet locomotion in oval squid Sepioteuthis lessoniana. Journal of Experimental Biology 209, 433-443.

Ward, D.V., 1972. Locomotory function of the squid mantle. Journal of Zoology 167, 487-499.

Weihs, D., 1974. Energetic advantages of burst swimming of fish. Journal of Theoretical Biology 48, 215-229.

Weymouth, G.D., Subramaniam, V., Triantafyllou, M.S., 2015. Ultra-fast escape maneuver of an octopus-inspired robot. Bioinspiration \& biomimetics 10, 016016.

Weymouth, G.D., Triantafyllou, M.S., 2013. Ultra-fast escape of a deformable jet-propelled body. J

Fluid Mech 721, 367-385.

Wilcox, D.C., 1998. Turbulence modeling for CFD. DCW industries La Canada, CA. 
Xiao, Q., Liao, W., 2010. Numerical investigation of angle of attack profile on propulsion performance of an oscillating foil. Comput Fluids 39, 1366-1380.

Xiao, Q., Liao, W., Yang, S., Peng, Y., 2012. How motion trajectory affects energy extraction performance of a biomimic energy generator with an oscillating foil? Renewable energy 37, 61-75. 